\title{
ACOUSTIC SIGNATURES IN THE COSMIC MICROWAVE BACKGROUND
}

\author{
WAYNE Hu \\ Institute for Advanced Study, School of Natural Sciences, Princeton, NJ 08540; whu@sns.ias.edu \\ AND \\ MARTIN WHITE \\ Enrico Fermi Institute, University of Chicago, Chicago, IL 60637; white@oddjob.uchicago.edu \\ Received 1996 February 5; accepted 1996 May 31
}

\begin{abstract}
We study the uniqueness and robustness of acoustic signatures in the cosmic microwave background by allowing for the possibility that they are generated by some as yet unknown source of gravitational perturbations. The acoustic pattern of peak locations and relative heights predicted by the standard inflationary cold dark matter model is essentially unique, and its confirmation would have deep implications for the causal structure of the early universe. A generic pattern for isocurvature initial conditions arises due to backreaction effects but is not robust to exotic source behavior inside the horizon. If present, the acoustic pattern contains unambiguous information on the curvature of the universe even in the general case. By classifying the behavior of the unknown source, we determine the minimal observations necessary for robust constraints on the curvature. The diffusion damping scale provides an entirely modelindependent cornerstone upon which to build such a measurement. The peak spacing, if regular, supplies a precision test.
\end{abstract}

Subject headings: cosmic microwave background - cosmology: theory

\section{INTRODUCTION}

Cosmic microwave background (CMB) anisotropies provide a unique window into the early universe through which we receive information on both the model for structure formation in the universe (e.g., Efstathiou, Bond, \& White 1992; Gorski et al. 1995; Ostriker \& Steinhardt 1995; Crittenden \& Turok 1995; Albrecht et al. 1996; Durrer, Gangui, \& Sakellariadou 1996; Dodelson, Gates, \& Stebbins 1995) and the background cosmology (e.g., Bond et al. 1994; Hu, Sugiyama, \& Silk 1996; Jungman et al. 1995; Seljak 1994; Scott \& White 1995). In the simplest models for structure formation, based on the gravitational instability of initial density perturbations, the acoustic signature in the anisotropy power spectrum provides a clean and unambiguous means of measuring all of the parameters of a Friedman-Robertson-Walker cosmology. In particular, it offers a standard ruler with which to make a classical test for curvature in the universe (Doroshkevich, Zel'dovich, \& Sunyaev 1978; Sugiyama \& Gouda 1992; Kamionkowski, Spergel \& Sugiyama 1994; Hu \& White 1996). However, structure formation may have proceeded by a more complicated route. Recent investigations have begun to probe the acoustic signature in texture (Crittenden \& Turok 1995; Durrer et al. 1996) and string (Albrecht et al. 1996; Magueijo et al. 1996) models for structure formation. Their predictions differ strongly from the standard inflationary case and suggest that perhaps without prior knowledge of the correct model for structure formation, the information contained in the acoustic signature cannot be extracted. In this paper we focus on two general questions: does the acoustic signature uniquely specify the model for structure formation? how robust is a measurement of the curvature to changes in the underlying model?

As discussed in the Appendix, the question of uniqueness is especially interesting in the case of the standard inflationary paradigm. Inflation is the unique causal mechanism for generating correlated curvature perturbations above the horizon (Liddle 1995). All other causal mechanisms gener- ate significant curvature perturbations only near horizon crossing. We will refer to these alternate possibilities as isocurvature models. A unique signature of superhorizon curvature fluctuations can be used as a test of inflation. By generalizing the external source formalism of $\mathrm{Hu} \&$ Sugiyama (1995a and 1995b, hereafter HSa and HSb) to include backreaction effects in $\S 2$, we find that a large class of isocurvature models carry a distinct acoustic signature that can be easily distinguished from the inflationary case, independent of the curvature and other cosmological parameters. The distinction lies in the gross properties of the spectrum, not small or subtle shifts in the peaks heights that require very high resolution measurements to see. As shown in $\S 3$, tell-tale features include the harmonic series of peak locations, the alternating relative peak heights due to baryon drag, and the diffusion damping tail. From this study, we conclude in $\S 4$ that the standard inflationary model with big bang nucleosynthesis (BBN) baryon-to-photon ratio bears an essentially unique signature.

If acoustic oscillations are present in the CMB, as is the case in all but models with significant reionization (see, e.g., Peebles 1987; Efstathiou \& Bond 1987; Coulson et al. 1994) or late formation of perturbations (Jaffe, Stebbins, \& Frieman 1994), their signature will provide information on the curvature. The damping tail contains the most robust information, but its location alone can constrain but not precisely fix the curvature. Additional restrictions such as a standard recombination history and a near BBN baryon content are required to make this a sensitive probe of the curvature. Furthermore, in models where the acoustic signature is sufficiently regular, the spacing of the peaks provides a precision test of the curvature even if the baryon content is anomalously low or somewhat high. It can also be combined with the damping tail to discriminate against truly exotic models. In $\S 5$, we discuss specifically what regularities must be observed before the curvature can be unambiguously measured if the correct model for structure formation is not assumed to be known a priori. 
The outline of this paper is as follows. The next section contains the derivation of our principle results. Their impact on the features in the angular power spectrum of the CMB is described in $\S 3$. We discuss our main points in $\S \S 4$ and 5 , and we present our conclusions in $\S 6$.

\section{PHYSICAL PROCESSES}

This section develops the formalism behind our principle results and illustrates them in a series of concrete examples. The lessons learned here will be applied in the later sections. A summary of important conclusions is given in $\S 2.9$, so that the bulk of this section may be skimmed on a first reading.

Acoustic oscillations in the CMB are inevitable if gravitational potential perturbations exist during the period when the Compton mean free path of a photon scattering off a free electron is much less than the horizon scale. In this case, the photons and electrons are tightly coupled. Since Coulomb interactions couple the electrons to the baryons, we refer to the system as a photon-baryon fluid. Photon pressure in the fluid resists gravitational compression and sets up acoustic waves in the system.

Because the properties of the oscillator are determined by the background, while those of the gravitational forces are described by the model for the perturbations, the acoustic signature provides a unique opportunity to probe both the background cosmology and the model for structure formation. Here, we explore the evolution of acoustic phenomena under the influence of an arbitrary source of gravitational perturbations. This is employed in $\S 3$ to determine the conditions under which signatures such as the peak locations, relative heights, and damping tail may be considered robust. These signatures will be observable in the smallscale CMB anisotropy if the tight coupling condition is satisfied during the epoch immediately preceding the last scattering event. In particular, it holds for the standard thermal history where recombination and hence last scattering occurs at redshift $z_{*} \sim 10^{3}$

\subsection{Fluid Equations}

We start with the fundamental equations describing the dynamics of a relativistic fluid. The physical interpretation and description of these equations is given below. The evolution of the photons and baryons in a metric perturbed by density fluctuations in the $k$ th normal mode is given in the Newtonian representation as (see, e.g., Mukhanov, Feldman, \& Brandenberger 1992; Ma \& Bertschinger 1995)

$$
\begin{gathered}
\dot{\Theta}_{0}=-\frac{k}{3} \Theta_{1}-\dot{\Phi}, \\
\dot{\delta}_{b}=-k V_{b}-3 \dot{\Phi},
\end{gathered}
$$

for the continuity equations and

$$
\begin{gathered}
\dot{\Theta}_{1}=k\left(\Theta_{0}+\Psi-\frac{1}{6} \Pi_{\gamma}\right)-\dot{\tau}\left(\Theta_{1}-V_{b}\right), \\
\dot{V}_{b}=-\frac{\dot{a}}{a} V_{b}+k \Psi+\dot{\tau}\left(\Theta_{1}-V_{b}\right) / R,
\end{gathered}
$$

for the momentum conservation or Euler equations of the photons and baryons, respectively. Here overdots are derivatives with respect to conformal time $\eta=\int d t / a, R=$ $3 \rho_{b} / 4 \rho_{\gamma}$ is the baryon-photon momentum density ratio, and $\dot{\tau}=x_{e} n_{e} \sigma_{\mathrm{T}} a$ is the differential Compton optical depth with $x_{e}$ as the ionization fraction, $n_{e}$ as the electron number density, and $\sigma_{\mathrm{T}}$ as the Thomson cross section. The fluctuations are defined as $\Theta_{0}=\Delta T / T=\delta_{\gamma} / 4$ the isotropic temperature perturbation, $\Theta_{1}$ the dipole moment or photon bulk velocity, $\Pi_{\gamma}$ the photon anisotropic stress perturbation, $\delta_{b}$ the baryon energy density perturbation, and $V_{b}$ the baryon velocity. The gravitational sources are $\Phi$, the perturbation to the spatial curvature, and $\Psi$, the Newtonian potential. In this gauge, these gravitational perturbations distort the metric as $g_{00}=-a^{2}(1+2 \Psi Q)$ and $g_{i j}=$ $a^{2}(1+2 \Phi Q) \gamma_{i j}$, where $\gamma_{i j}$ is the three metric on a surface of constant curvature and $Q$ is a plane wave $\exp (i \boldsymbol{k} \cdot \boldsymbol{x})$ in a flat geometry or more generally the $k$-eigenfunction of the Laplacian (Wilson 1983). The Einstein-Poisson equations thus relate them to the matter fluctuations as (Bardeen 1980)

$$
\begin{aligned}
\left(k^{2}-3 K\right) \Phi & =4 \pi G a^{2} \sum\left[\rho_{i} \delta_{i}+3 \frac{\dot{a}}{a}\left(\rho_{i}+p_{i}\right) \frac{V_{i}}{k}\right], \\
k^{2}(\Psi+\Phi) & =-8 \pi G a^{2} \sum p_{i} \Pi_{i},
\end{aligned}
$$

where the sum is over particle species, the curvature $K=$ $-H_{0}^{2}\left(1-\Omega_{0}-\Omega_{\Lambda}\right)$, and the Hubble constant $H_{0}=100 h$ $\mathrm{km} \mathrm{s}^{-1} \mathrm{Mpc}^{-1}$. We will assume from now on that the relevant scales are far under the curvature scale $\left(K / k^{2} \rightarrow 0\right)$. None of our main results are affected by this assumption for reasonable values of $K$. Note also that if the anisotropic stress $p_{T} \Pi_{T}=\sum p_{i} \Pi_{i}$ is negligible, $\Psi=-\Phi$.

Now let us examine the physical content of equations (1) and (2). Photon number conservation relates changes in the temperature fluctuations to the velocity divergence by a factor of $\frac{1}{3}$ (since number density is related to temperature by $n_{\gamma} \sim T^{3}$ ). The $\dot{\Phi}$ term represents the dilation effect on the wavelength of the photons. Since the curvature perturbation "stretches" the spatial metric, changes in $\Phi$ gives rise to a dilation effect of the same origin as the cosmological redshift. Notice the sign of this effect implies that the photons will always oppose a change in the curvature, a point that will be very important later. Similar effects govern the baryon continuity equation. Since the fractional energy and number density fluctuations are equal for a nonrelativistic particle, their rate of change is given by the velocity divergence. The additional effect due to the stretching of the volume from $\dot{\Phi}$ also implies a number density dilution of $3 \dot{\Phi}$.

The expansion makes particle momenta decay as $a^{-1}$. For the photons, this is accounted for by the temperature redshift; for the baryons, by expansion drag on the bulk velocity (the $\dot{a} / a$ term). Gradients in the potential, $k \Psi$, generate velocity perturbations by gravitational infall. For the photons, infall is countered by stress in the fluid, both isotropic (pressure) and anisotropic (quadrupole moment). The baryons, however, are effectively pressureless. The photon and baryon equations are coupled by Compton scattering (the $\dot{\tau}$ terms), which exchanges momentum between the fluids. Since the momentum density of the fluid is proportional to $\rho+p$, conservation relates the scattering terms by the factor $R=\left(p_{b}+\rho_{b}\right) /\left(p_{\gamma}+\rho_{\gamma}\right) \approx 3 \rho_{b} / 4 \rho_{\gamma}$. Scattering seeks to equalize the bulk velocities $\Theta_{1}=V_{b}$ causing adiabatic evolution of the density perturbations $\dot{\delta}_{b}=3 \dot{\Theta}_{0}$.

If the scattering is rapid compared with the travel time across a wavelength, the momentum conservation equation (2) may be expanded in powers of the Compton mean free path over the wavelength $k / \tau$. By eliminating the baryon velocity, we obtain the tight coupling approximation for the 
evolution of the photons (Peebles \& Yu 1970; HSa):

$$
\begin{aligned}
& \dot{\Theta}_{0}=-\frac{k}{3} \Theta_{1}-\dot{\Phi}, \\
& \dot{\Theta}_{1}=-\frac{R}{1+R} \frac{\dot{a}}{a} \Theta_{1}+\frac{1}{1+R} k \Theta_{0}+k \Psi .
\end{aligned}
$$

The quadrupole term $\Pi_{y}=\mathcal{O}(k / \dot{\tau}) \Theta_{1}$ causes viscous damping and is treated in $\S 2.8$. It is a higher order correction because scattering tends to isotropize the photons in the baryon rest frame and suppresses the quadrupole. From examining equation (4), one can see that baryons decrease the efficacy of the pressure and add an expansion drag term to the momentum equation. The gravitational infall term remains unaltered since its baryon analog is identical.

\subsection{Gravitational Redshift Effects}

Before proceeding with the main task of exploring the acoustic signatures, let us review how gravitational effects manifest themselves in the CMB (Sachs \& Wolfe 1967). As discussed in $\S 2.1$, these are the ordinary redshift of a photon climbing out in and out of potential wells and the dilation effect from changes in the spatial metric. If the metric fluctuations are generated by the density fluctuations in the photon-baryon system itself, the Poisson equations (eq. [3]) tell us that they are suppressed by a factor of $(k \eta)^{-2}$ with respect to the temperature fluctuations inside the horizon. Thus, the self-gravity of the photon-baryon fluctuations generally only is important outside the horizon $k \eta \ll 1$. However, this is not necessarily true for metric fluctuations generated by an external source. Gravitational redshift effects can significantly alter the acoustic signature, and we must include them in the analysis even on small scales.

Because temperature perturbations are observed only after the photons have lost energy climbing out of potential wells $\Psi$, the "effective" temperature perturbation is given by $\Theta_{0}+\Psi$. It is this quantity that we measure as a temperature fluctuation on the sky. It will be important in the following sections to consider the effective temperature fluctuation rather than the intrinsic fluctuation $\Theta_{0}$. The blueshift from infall into a constant gravitational well is exactly canceled by the redshift from climbing out. Thus, $\Theta_{0}$ may have a large but unobservable offset, which is removed in the effective temperature $\Theta_{0}+\Psi$.

On the other hand, the intrinsic temperature evolves as $\dot{\Theta}_{0}=-\dot{\Phi}$ above the horizon to oppose changes in the spatial curvature. Thus, the effective temperature obeys the relation $\dot{\Theta}_{0}+\dot{\Psi}=\dot{\Psi}-\dot{\Phi} \approx-2 \dot{\Phi}$ above the horizon. This yields a general description of the gravitational redshift or Sachs-Wolfe effect (Sachs \& Wolfe 1967),

$$
\left[\Theta_{0}+\Psi\right](\eta, k)=\left[\Theta_{0}+\Psi\right]\left(\eta_{i}, k\right)+\left.[\Psi-\Phi](\eta, k)\right|_{\eta_{i}} ^{\eta},
$$

where $\eta_{i}$ is some initial epoch at which the fluctuations were formed. In particular, if the initial conditions are isocurvature $\Phi\left(\eta_{i}, k\right)=0$, and the initial temperature fluctuation is also small, then the effective temperature becomes $\Psi-\Phi \approx-2 \Phi$. The photons are thus underdense in potential wells. For adiabatic fluctuations, photons are overdense in potential wells so that the effective temperature fluctuation is reduced. As we shall see below, in the radiation-dominated era $\Theta_{0}+\Psi=\frac{1}{2} \Psi$. The change in the equation of state through the matter-radiation transition causes a small decay in the potential (see Appendix, eq.
[A4]) and brings the effective temperature in the matterdominated limit to $\Theta_{0}+\Psi=\frac{1}{3} \Psi$ (Sachs \& Wolfe 1967; $\mathrm{HSa})$.

\subsection{Oscillator Equation}

In $\mathrm{HSa}$, a formalism was developed to calculate the response of the photon-baryon fluid to metric fluctuations $\Psi$ and $\Phi$, which are considered external to the fluid. Combining the two equations in equation (4), we obtain

$$
\frac{d}{d \eta}(1+R) \dot{\Theta}_{0}+\frac{k^{2}}{3} \Theta_{0}=-\frac{k^{2}}{3}(1+R) \Psi-\frac{d}{d \eta}(1+R) \dot{\Phi} .
$$

Conceptually, this equation reads: the change in momentum of the photon baryon fluid is determined by a competition between the pressure restoring and the gravitational driving forces. Below the sound horizon,

$$
r_{s}(\eta)=\int_{0}^{\eta} d \eta^{\prime} c_{s}=\int_{0}^{\eta} d \eta^{\prime} \frac{1}{\sqrt{3(1+R)}},
$$

photon pressure resists gravitational compression and sets up acoustic oscillations. Though conceptually useful, this approach has the practical disadvantage that the photonbaryon contribution to the metric fluctuations must be already known. In general, it is unknown and we must break the metric fluctuations up into pieces generated by the photon-baryon fluid $(\gamma b)$ and by the external source $(s)$, e.g., from dark matter, entropy fluctuations, defects, etc. The Poisson equations (eq. [3]) and the tight coupling condition $V_{b}=\Theta_{1}$ tell us

$$
\begin{aligned}
\Phi_{b \gamma} & =-\Psi_{b \gamma} \\
& =6 \Omega_{\gamma} H_{0}^{2}(a k)^{-2}(1+R)\left[\Theta_{0}+\frac{\dot{a}}{a} \frac{1}{k} \Theta_{1}\right],
\end{aligned}
$$

for adiabatic fluctuations in the photon-baryon system where $\delta_{b}=3 \Theta_{0}$. As we shall see in $\S 2.5$, entropy fluctuations $S=\delta_{b}-3 \Theta_{0}$ are simply described as an external source since $\dot{S}=0$ in the tight coupling limit. Thus, equation (8) represents no loss of essential generality.

As equation (8) implies, the response of the photonbaryon fluid is most easily solved in the so-called rest frame of the fluid (see Appendix). Here the temperature perturbation is

$$
\mathscr{T}=\Theta_{0}+\frac{\dot{a}}{a} \frac{1}{k} \Theta_{1} .
$$

Combining equations (4) and (9), it evolves under the equations

$$
\begin{aligned}
& {\left[1+\frac{6}{y^{2}}(1+R)\right]\left(\mathscr{T}^{\prime}-\frac{y^{\prime}}{y} \frac{1}{1+R} \mathscr{T}\right)} \\
& +\frac{1}{3}\left[1-3 \frac{y^{\prime \prime}}{y}+6\left(\frac{y^{\prime}}{y}\right)^{2}\right] \Theta_{1}=\frac{y^{\prime}}{y} \Psi_{s}-\Phi_{s}^{\prime}, \\
& \Theta_{1}^{\prime}+\frac{y^{\prime}}{y} \Theta_{1}-\left[1-\frac{6}{y^{2}}(1+R)^{2}\right] \frac{1}{1+R} \mathscr{T}=\Psi_{s},
\end{aligned}
$$

where primes are derivatives with respect to $x=k \eta$ with $k$ fixed, and $y=\left(\Omega_{\gamma} H_{0}^{2}\right)^{-1 / 2} a k$. Notice that if $\left|\Theta_{1}\right| \lesssim\left|\Theta_{0}\right|$, as is the case in the oscillatory regime $x c_{s} \gtrsim 1$, equation (9) implies that $\mathscr{T}=\Theta_{0}\left[1+\mathcal{O}\left(x^{-1}\right)\right]$ and the two representa- 
tions become equivalent. In the next few sections, we will examine the implications of equation (10) through a number of examples and approximations.

\subsection{Photon Backreaction at Early Times}

The evolution equation (10) simplifies substantially in the radiation-dominated epoch. Early on, the baryon contribution is negligible and the fluid evolution is dominated by the response of the photon perturbations to the source. The resulting photon-baryon density fluctuation feeds back into the metric fluctuation. Even though the universe may be just becoming matter dominated at last scattering, its early radiation-dominated legacy plays the dominant role. As we shall see, the processes that fix the amplitude of the acoustic oscillation take effect mainly around horizon crossing when the universe was still radiation dominated for the relevant fluctuations.

It is instructive to consider first the case in which the expansion is photon-dominated. This neglects the neutrino and source contribution to the background energy density but does not fundamentally alter the results for the early superhorizon evolution (see $\mathrm{HSb}$ ). In this limit, $R \rightarrow 0$, $y \rightarrow x$, and the evolution equations (10) become

$$
\begin{gathered}
\mathscr{T}^{\prime}-\frac{\mathscr{T}}{x}+\frac{\Theta_{1}}{3}=\frac{x^{2}}{x^{2}+6}\left[\frac{\Psi_{s}}{x}-\Phi_{s}^{\prime}\right], \\
\Theta_{1}^{\prime}+\frac{\Theta_{1}}{x}-\left[1-\frac{6}{x^{2}}\right] \mathscr{T}=\Psi_{s},
\end{gathered}
$$

or combining the two,

$$
\mathscr{T}^{\prime \prime}+\frac{1}{3}\left(1-\frac{6}{x^{2}}\right) \mathscr{T}=\mathscr{S},
$$

where the gravitational source is given by

$$
\begin{aligned}
\mathscr{S}= & -\left[\frac{1}{3}-\frac{12}{\left(x^{2}+6\right)^{2}}\right] \Psi_{s}-\frac{x\left(x^{2}+18\right)}{\left(x^{2}+6\right)^{2}} \Phi_{s}^{\prime} \\
& +\frac{x}{x^{2}+6} \Psi_{s}^{\prime}-\frac{x^{2}}{x^{2}+6} \Phi_{s}^{\prime \prime} .
\end{aligned}
$$

If $\mathscr{S}=0$, this is simply the Bessel equation. The homogeneous solutions are therefore (Kodama \& Sasaki 1986)

$$
\begin{aligned}
& \mathscr{T}_{a}(x)=-\cos (x / \sqrt{3})+(\sqrt{3} / x) \sin (x / \sqrt{3}), \\
& \mathscr{T}_{b}(x)=-\sin (x / \sqrt{3})-(\sqrt{3} / x) \cos (x / \sqrt{3}),
\end{aligned}
$$

with Wronskian $3^{-1 / 2}$. These functions are plotted in Figure 1 and may be considered as the fundamental or pure modes of the photon-baryon fluid. Their limiting behavior as $x \rightarrow 0$ is $\mathscr{T}_{a}=x^{2} / 9$ and $\mathscr{T}_{b}=-3^{1 / 2} / x$. From equation (9), the corresponding limits for the Newtonian fluctuations are $\Theta_{0}=1 / 3$ and $6(3)^{1 / 2} / x^{3}$ and $\Phi_{\gamma b}=2 / 3$ and $-6(3)^{1 / 2} / x^{3}$ for the two modes, respectively. As $x \rightarrow \infty$, they become cosine and sine waves, respectively, for both the rest frame and Newtonian temperatures.

Although equation (11) may easily be solved numerically, Green's method is more illuminating. Constructing the solution out of the pure modes, we find

$$
\mathscr{T}(x)=\mathscr{A}_{a}(x) \mathscr{T}_{a}(x)+\mathscr{A}_{b}(x) \mathscr{T}_{b}(x),
$$

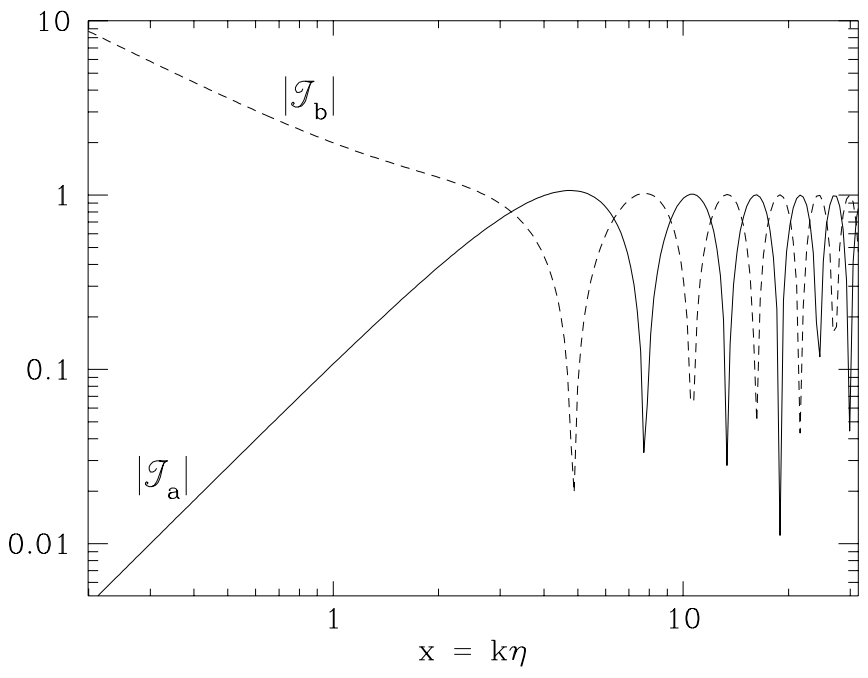

FIG. 1.-Pure modes. In the absence of sources, the growing mode of the rest frame temperature perturbation matches onto a cosine acoustic oscillation inside the horizon, whereas the decaying mode matches onto a sine oscillation. The oscillator response to an external source is constructed by Greens method from these homogeneous solutions and transformed into the Newtonian frame with eq. (9).

with

$$
\begin{aligned}
& \mathscr{A}_{a}(x)=\mathscr{A}_{a}\left(x_{i}\right)-\sqrt{3} \int_{x_{i}}^{x} \mathscr{T}_{b}\left(x^{\prime}\right) \mathscr{S}\left(x^{\prime}\right) d x^{\prime}, \\
& \mathscr{A}_{b}(x)=\mathscr{A}_{b}\left(x_{i}\right)-\sqrt{3} \int_{x_{i}}^{x} \mathscr{T}_{a}\left(x^{\prime}\right) \mathscr{S}\left(x^{\prime}\right) d x^{\prime},
\end{aligned}
$$

where $x_{i}$ is the initial epoch at which the perturbations, and hence, the constants $\mathscr{A}_{a}\left(x_{i}\right)$ and $\mathscr{A}_{b}\left(x_{i}\right)$ are fixed. If the photon fluctuations are set to zero at $x_{i}$, then the answer can be read directly off the source behavior. When $\mathscr{T}_{b} \gg$ $\mathscr{T}_{a}$, source contributions stimulate the $\mathscr{T}_{a}$ mode mainly. An examination of Figure 1 would imply that all superhorizon effects from the source would create a $\mathscr{T}_{a}$ cosine mode. However, the initial fluctuations could be set up such that $\mathscr{A}_{a}\left(x_{i}\right)$ and $\mathscr{A}_{b}\left(x_{i}\right)$ exactly cancel the influence of the source. This is exactly what occurs in the class of isocurvature models based on balanced initial conditions.

To see this more clearly, let us express the evolution in terms of $\Phi_{\gamma b}$, the curvature perturbation generated by the photon-baryon fluid. In the photon-dominated limit, equation (8) gives $\Phi_{\gamma b}=6 \mathscr{T} / x^{2}$ and equation (12) becomes

$$
x^{2} \Phi_{\gamma b}^{\prime \prime}+4 x \Phi_{\gamma b}^{\prime}=-x^{2} \Phi_{s}^{\prime \prime}-4 x \Phi_{s}^{\prime}
$$

for $x \ll 1$. The solution is

$$
\begin{aligned}
\Phi(x)= & \Phi_{\gamma b}(x)+\Phi_{s}(x) \\
= & \Phi_{\gamma b}\left(x_{i}\right)+\Phi_{s}\left(x_{i}\right)+\frac{1}{3}\left[\Phi_{\gamma b}+\Phi_{s}\right. \\
& \left.+\left(2 / x_{i}\right) \Theta_{1}\right]\left(x_{i}\right)\left[\left(x_{i} / x\right)^{3}-1\right],
\end{aligned}
$$

where we have approximated $\Psi_{s}\left(x_{i}\right)=-\Phi_{s}\left(x_{i}\right)$ in rewriting the initial conditions in terms of $\Theta_{1}$. Aside from a decaying mode, the photons evolve to keep the curvature perturbation constant (cf. eq. [A9] and Veeraraghavan \& Stebbins 1990). Thus, independent of the source behavior, if $\Phi_{b \gamma}\left(x_{i}\right)=-\Phi_{s}\left(x_{i}\right)$ and $\Theta_{1}\left(x_{i}\right)=0$, compensation forces $\Phi(x \ll 1) \approx 0$.

While this argument only shows compensation for a photon-dominated system, the argument applies equally 
well for whatever the dominant dynamical component is since $\dot{\delta} \propto-\dot{\Phi}$ for $x \rightarrow 0$. The argument does not strictly apply if the identity of this component, i.e., the equation of state, changes. For example, pressure fluctuations and hypersurface warping can change the curvature through the matter-radiation transition even if $x \ll 1$. We discuss these points further in the Appendix and show that densities and hence temperature fluctuations are anticorrelated with the total curvature outside the horizon for isocurvature initial conditions (see also eq. [5]).

\subsection{Driven Oscillations and Superhorizon Effects}

In this section we will see how superhorizon compensation drives the oscillator and stimulates one of the two pure modes of $\S 2.4$. Let us start with a simple and concrete example: the baryon isocurvature case. Here, we begin at some initial epoch with an entropy fluctuation $S\left(x_{i}\right)=$ $\delta\left(n_{b} / n_{\gamma}\right)=\delta_{b}\left(x_{i}\right)-3 \Theta_{0}\left(x_{i}\right)$. Tight coupling implies that the number density fluctuation of the photons and baryons, and hence $S$, remains constant (see eq. [1]). The entropy acts as an external source, which from equation (3) contributes as $k^{2} \Phi_{s}=4 \pi G a^{2} \rho_{b} S$, or, rewriting this in the form of equation (8),

$$
\Phi_{s}=-\Psi_{s}=\frac{2}{x^{2}} R S=\frac{A}{x},
$$

where $A=(3 / 2)\left(\Omega_{b} H_{0}^{2}\right)\left(\Omega_{\gamma} H_{0}^{2}\right)^{-1 / 2} k^{-1} S$. Notice that the curvature perturbation implied by the source actually increases as $x \rightarrow 0$. For this model, the effective source in equation (13) reduces to the simple form:

$$
\mathscr{S}=-\frac{1}{3} \Psi_{s}=\frac{A}{3} \frac{1}{x} .
$$

Now let us assume the isocurvature condition at the initial epoch $\Phi\left(x_{i}\right)=0$, or $\Phi_{b \gamma}\left(x_{i}\right)=-\Phi_{s}\left(x_{i}\right)$ and $\Theta_{1}\left(x_{i}\right)=0$ (see also Appendix). By requiring continuity in $\mathscr{T}$ and its first derivative, the initial partition into pure modes in equation (16) becomes $\mathscr{A}_{a}\left(x_{i}\right)=-A / x_{i}$ and $\mathscr{A}_{b}\left(x_{i}\right)=\left(3^{1 / 2} / 54\right) A x_{i}^{2}$, yielding a large contribution to the $\mathscr{T}_{a}$ mode. However, let us examine the influence of the source term on the sub- sequent evolution:

$$
\begin{aligned}
& \sqrt{3} \int_{x_{i}}^{x} \mathscr{T}_{a}\left(x^{\prime}\right) \mathscr{S}\left(x^{\prime}\right) d x^{\prime}=-(\sqrt{3} / 54)\left(x^{2}-x_{i}^{2}\right) A, \\
& \sqrt{3} \int_{x_{i}}^{x} \mathscr{T}_{b}\left(x^{\prime}\right) \mathscr{S}\left(x^{\prime}\right) d x^{\prime}=\left(x^{-1}-x_{i}^{-1}\right) A, \quad(x \ll 1) .
\end{aligned}
$$

Thus, as the evolution progresses, the initially large $\mathscr{T}_{a}$ contribution drops precipitously, leaving mainly the $\mathscr{T}_{b}$ or sine mode at subhorizon scales $x \gtrsim 1$. Fundamentally, this is due to the feedback effect of equation (17): the photons oppose any change to the net curvature and evolve to maintain the isocurvature condition. In Figure 2, we show the time evolution of $\mathscr{T}$, the source curvature, and the total curvature in the baryon isocurvature model. By comparing $\Phi$ to $\Phi_{s}$, notice that the feedback effect is only important outside the horizon. Another interesting quantity is the ratio of the $\mathscr{T}_{a}$ to $\mathscr{T}_{b}$ amplitude shown in Figure $2 b$. In this case, the $\mathscr{T}_{a}$ mode essentially disappears after horizon crossing leaving the acoustic perturbation in a pure sine mode $(p=-1.0$, heavy line). In fact, the integral in equation (16) takes on a simple asymptotic form (HSb):

$$
\begin{aligned}
& \lim _{x \rightarrow \infty} \mathscr{A}_{a}(x)=0, \\
& \lim _{x \rightarrow \infty} \mathscr{A}_{b}(x)=A / \sqrt{3} .
\end{aligned}
$$

We also show in Figure $2 b$ the behavior when the source is generalized to $\Phi_{s}=-\Psi_{s}=A x^{p}$. Again, the initially large $\mathscr{T}_{a}$ mode is reduced as the superhorizon scale evolution progresses.

Even if the source has some superhorizon scale feature, i.e., a maximum at $x \ll 1$, its effect is mainly canceled out. From equation (18), notice that the photons always attempt to counter the source. The photon fluctuations would track the rise and fall of a feature leaving an effect only from the boundary conditions. Below the horizon, however, compensation cannot occur due to the intervention of photon pressure.

How then does one obtain strong contributions to the $\mathscr{T}_{a}$ cosine mode? If superhorizon fluctuations are not fully
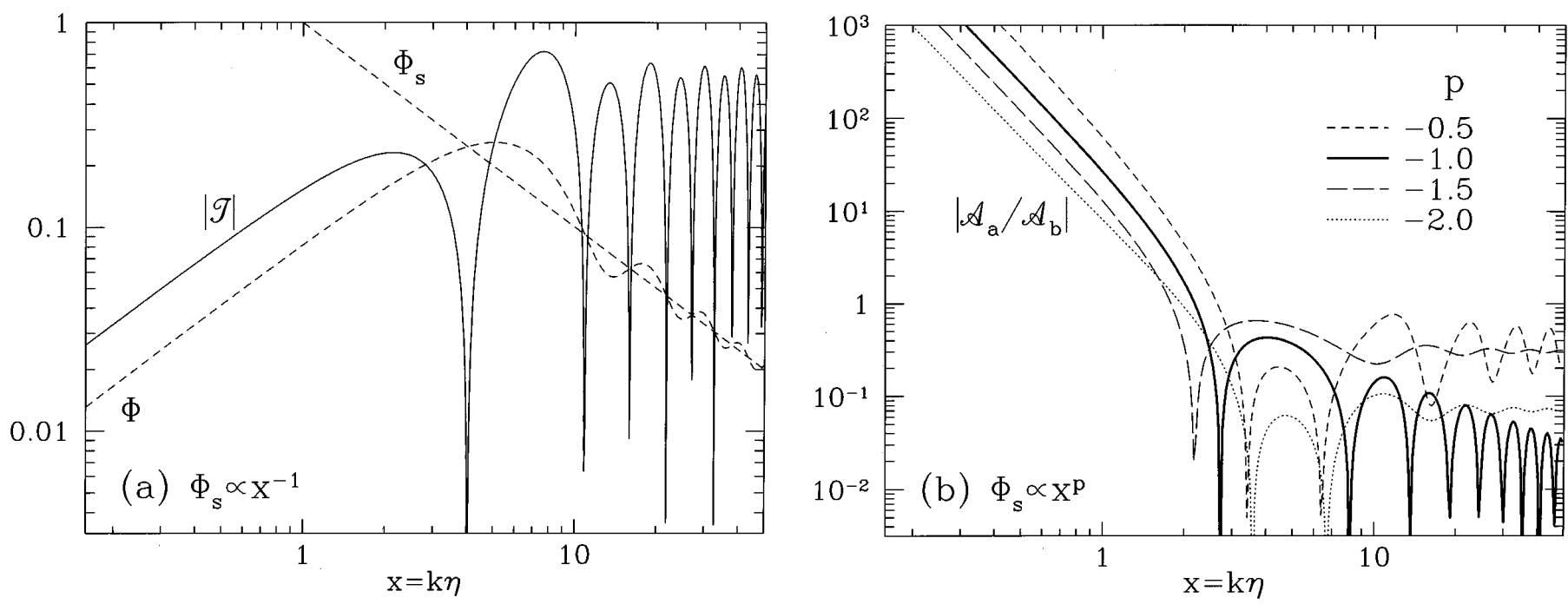

FIG. 2.-Compensation and isocurvature fluctuations. (a) Baryon isocurvature model $\left(\Phi_{s} \propto x^{-1}\right)$. Outside the horizon $x \lesssim 1$, backreaction from the photons cancels the contribution of the source to the curvature fluctuation. Inside the horizon, pressure prevents significant metric contributions from the photon-baryon fluid and $\Phi \rightarrow \Phi_{s}$ (b) For source functions $\Phi_{s} \propto x^{p}$, the ratio of $\mathscr{T}_{a}$ to $\mathscr{T}_{b}$ amplitudes decreases due to feedback. This leaves the acoustic oscillation mainly in the sine mode at $x \gg 1$. 
compensated, equation (16) implies a large amplitude in $\mathscr{T}_{a}$. This adiabatic component implies superhorizon curvature perturbations initially: $\Phi\left(x_{i}\right) \neq 0$. The simplest example is the case of $\Phi_{s}=0$ and $\Phi_{\gamma b}\left(x_{i}\right) \neq 0$. In Figure 3, this case is contrasted with the baryon isocurvature case. Let us use these examples to gain further intuition about the feedback mechanism.

From Figure 3, we see that in the isocurvature case, compensation prevents a large curvature fluctuation from appearing outside of the horizon regardless of the source. However, photon pressure, which becomes important around sound horizon crossing, resists the accompanying rarefaction of the photon fluid due to dilation. At this point, the fluid turns around and begins falling into the potential wells of the source enhancing the curvature fluctuation by its self-gravity. Note the increase in the amplitude of the oscillation between the negative maximum and the positive maximum. As the photons resist further compression at the positive maximum, the self-gravity contribution to the potential fluctuation decays. This leaves the photon-baryon fluid in a highly compressed state and increases the amplitude of the acoustic oscillation. Thus, the self-gravity of the photon-baryon fluid essentially drives the oscillator. It provides a kick at each of the first two turning points to enhance the oscillation.

A similar analysis applies to adiabatic fluctuations. Here the initial curvature fluctuation is kept constant outside the horizon by photon backreaction. From $\S 2.1$ recall that the intrinsic temperature fluctuation and gravitational potential partially cancel in the effective temperature. From the $x \ll 1$ limit of equation (14), $\Theta_{0}+\Psi_{\gamma b}=\frac{1}{2} \Psi_{\gamma b}<0$. At horizon crossing, the fluid begins to compress itself due to its self-gravity and the effective temperature reverses sign. As pressure begins to stop the compression, the potential decays. Again the fluid is left in a highly compressed state, and self-gravity acts as a driving term. The dilation due to $\Phi$ (see discussion following eq. [3]) doubles the effect of infall from $\Psi$, so that the end amplitude is $\frac{1}{2} \Psi_{\gamma b}\left(x_{i}\right)-2 \Psi_{\gamma b}\left(x_{i}\right) \approx$ $-(3 / 2) \Psi_{\gamma b}$.

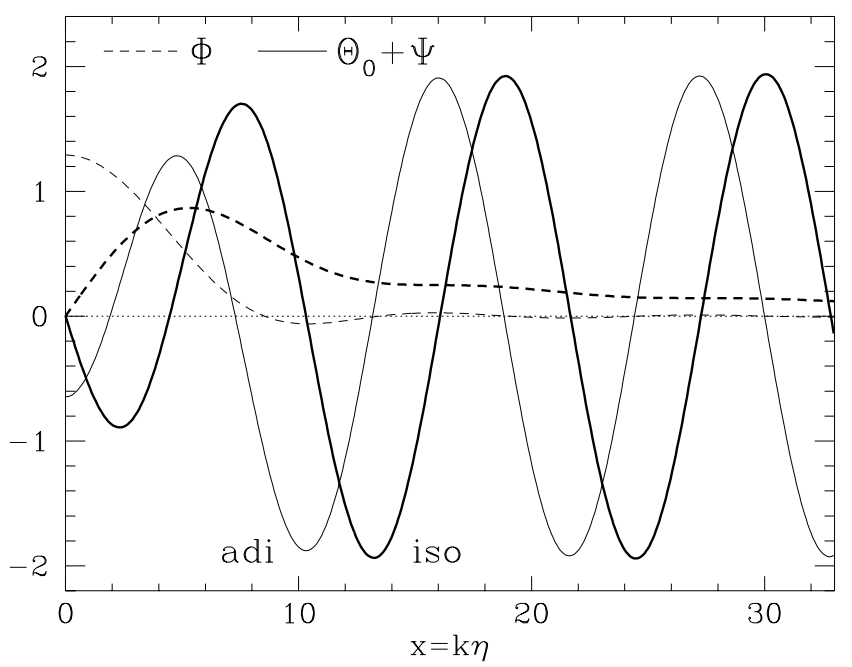

FIG. 3.-Driven oscillations. The self-gravity of the photon-baryon fluid drives a cosine oscillation for adiabatic initial conditions (thin lines) and a sine oscillation (thick lines) for isocurvature initial conditions. The adiabatic model has $\Phi_{s}\left(x_{i}\right)=0$ and $\Phi_{\gamma b}\left(x_{i}\right) \neq 0$. The isocurvature model is the baryonic model of eq. (19). The dashed lines show the full potential, the solid lines show the effective temperature. In both cases the amplitude in $\Theta_{0}+\Psi$ increase during the first few oscillations, as described in $\S 2.5$.
A difference in phase between the two modes arises since gravitational infall that initiates the chain of events leading to the adiabatic acoustic mode only takes effect inside the horizon, whereas the dilation effect that begins the isocurvature chain of events is already occurring outside the horizon. The end result is that the self-gravity of the photon-baryon fluid drives a cosine oscillation for adiabatic initial conditions and a sine mode for isocurvature initial conditions. This is not the most general case, however, since self-gravity and thus the initial conditions only dominate the behavior around or before horizon crossing. We consider the behavior after horizon crossing in the next section.

\subsection{Forced Oscillations and Subhorizon Effects}

If the source is still effective inside the horizon $\Phi_{s}(x>$ 1) $\geq \Phi_{s}(x=1)$, more complicated behavior can result since the regulatory mechanism of photon feedback is ineffective. This is the case of so-called "active perturbations" (Magueijo et al. 1996). The general Greens function solution can be rewritten as

$$
\begin{aligned}
\mathscr{T}(x)= & {\left[\mathscr{A}_{a}^{2}(x)+\mathscr{A}_{b}^{2}(x)\right]^{1 / 2} } \\
& \times \sin \left\{x / \sqrt{3}+\tan ^{-1}\left[\mathscr{A}_{a}(x) / \mathscr{A}_{b}(x)\right]\right\},
\end{aligned}
$$

for $x \gg 1$. The phase of the acoustic wave is in general time dependent for subhorizon effects. Furthermore, we cannot ignore the gravitational redshift of the photons climbing out of the potential even inside the horizon (see $\S 2.2$ ). The effective temperature is $\Theta_{0}+\Psi \approx \mathscr{T}+\Psi$ if $x \gg 1$. In Figure 4, we plot an example based on a broken power-law source of the form

$$
\Phi_{s}=\frac{1}{(\alpha x)^{-p_{1}}+x^{p_{2}}} .
$$

Here $p_{1}=3, p_{2}=1$ and $\alpha=0.01$ such that the source peaks well below the horizon scale. Initial conditions do not play a major role here since the fluctuation is generated inside the horizon. By considering only the effective temperature

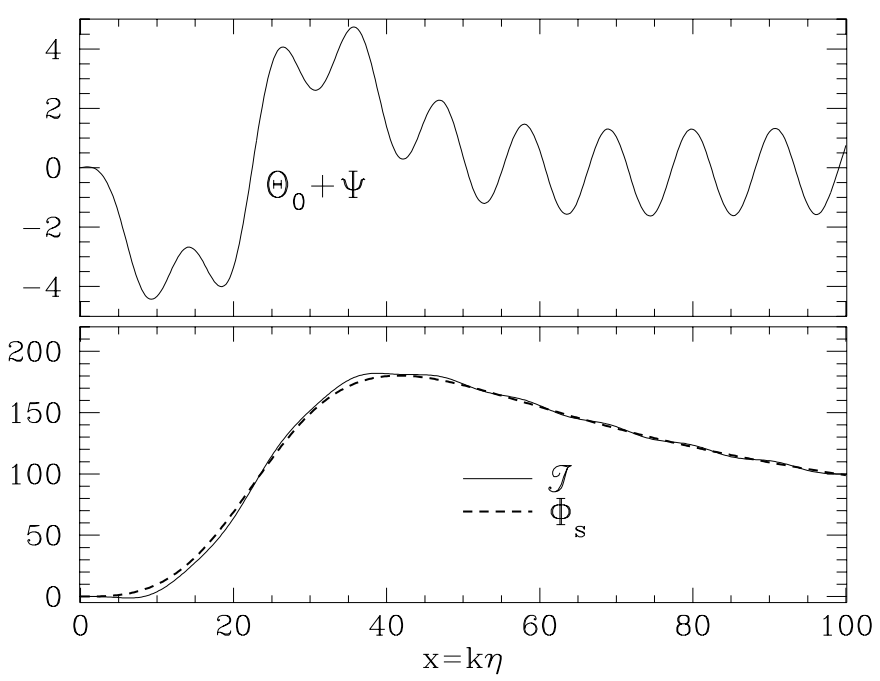

FIG. 4.-Forced oscillations. Inside the horizon, the regulatory effects of photon-baryon self-gravity become ineffective. A source that peaks at $x=k \eta \gg 1$ can produce complicated forced oscillations in the fluid. The intrinsic temperature fluctuation $\mathscr{T} \approx \Theta_{0}$ suffers a large unobservable offset $-\Psi_{s}$ (bottom panel), which is removed by the redshift in the effective temperature $\Theta_{0}+\Psi$ (top panel). Notice that the fundamental period $k r_{s}=$ $2 \pi$ or $x \simeq 10.9$ is still clearly apparent for this slowly varying source. 
$\Theta_{0}+\Psi$, we remove the large unobservable offset from gravitational blueshift in $\mathscr{T}$ (see $\S 2.2)$. Note that it is important to include the Sachs-Wolfe contribution to obtain a reliable measure of the temperature anisotropy at last scattering. This is especially true when the anisotropies are "sourced" such that metric fluctuations remain substantial inside the horizon, as in some defect models. Neglecting this effect, for example by use of the oft-employed synchronousgauge photon density, can lead to erroneous conclusions about the shape of the CMB anisotropy power spectrum. Should the potentials have significant time variability after last scattering, it is also necessary to include the ISW effect since this can lead to significant distortions of the peaks, notably the first peak.

Even this smooth, well-behaved source leads to complicated structure in the acoustic oscillation. Furthermore, after the source turns off and $\mathscr{A}_{a}$ and $\mathscr{A}_{b}$ become constant in time, the oscillator is left with a phase shift described by equation (23) that does not necessarily correspond to one of the pure modes. It is important to note that even in this case, the temperature perturbation is anticorrelated with the source fluctuation immediately after horizon crossing. This is due to compensation from feedback around horizon crossing and will be important for the question of robust distinctions between models. We have found that this anticorrelation persists even if the source changes sign outside the horizon or near horizon crossing.

It is of course impossible to quantify all the possibilities that arise from the arbitrary action of a source well inside the horizon. However, Figure 4 illustrates two general points. Large metric and correspondingly even larger density fluctuations are required for subhorizon forcing to dominate over driving effects at horizon crossing. Compare the vertical scales of the upper and lower panels of Figure 4. There are two effects that make forcing around horizon crossing easier than well inside the horizon. The first is that for $x \gg 1$ a large metric perturbation requires a very large density perturbation, since the Poisson equation (eq. [3]) gives $\Phi_{s}=\left(\rho_{s} / \rho_{T}\right) \mathcal{O}\left(\delta_{s} / x^{2}\right)$. Also, from the Euler equation, pressure perturbations are more important than metric perturbations at large $x$, once the artificial offset due to the $k \Psi$ term has been removed by considering the effective temperature. From equation (6), the effective temperature evolves according to

$$
\left(\Theta_{0}+\Psi\right)^{\prime \prime}+\frac{1}{3}\left(\Theta_{0}+\Psi\right)=\Psi^{\prime \prime}-\Phi^{\prime \prime} .
$$

The source is thus $\approx-2 \Phi^{\prime \prime}$, and if constant, produces a temperature shift of $6 \Phi^{\prime \prime}$. Exotic spectra of this type require extreme conditions. More plausible is a model whose forced effects dominate only around $x \approx 1$, where feedback effects still play some role. As we shall see in $\S 3.2$, this has consequences for the coherence of the resulting oscillations.

The second point is that in spite of the gravitational forcing the natural period of the oscillation, here $x=2(3)^{1 / 2} \pi$ is still apparent in the harmonic structure. If the source $\Phi^{\prime \prime}$ is slowly varying in time, so is the phase shift. In Figure 4, the spacing between the peaks is still regular and corresponds to the natural period despite the slowly varying offset. Thus, this acoustic signature is only lost if the metric fluctuations are both extremely large and rapidly varying inside the horizon.

If such an exotic spectrum is measured in the CMB, we would have concrete evidence that the mechanism responsible for structure formation requires new physics.
Until the spectrum is measured, however, we can only consider the broader implications. The possibility of driven subhorizon effects raises two questions that we will address in $\S \S 4$ and 5 . Are there any unique signatures of the pure cases that distinguish them from these more exotic scenarios? How does the existence of such exotic models affect our ability to measure quantities such as the curvature of the universe? To help answer these questions, we need to understand two additional acoustic effects that can provide clues to unraveling the spectrum.

\subsection{Baryon Drag}

Up until this point, we have neglected the effect of the baryons in the photon-baryon fluid. This is appropriate for the early evolution and reveals the qualitative structure of the acoustic signal even at recombination since $R\left(\eta_{*}\right)=$ $3 \rho_{b} /\left.4 \rho_{\gamma}\right|_{\eta^{*}} \approx 31.5 \Omega_{b} h^{2} \sim 30 \%$. Nevertheless, there is an important acoustic signature associated with the baryons if $\Omega_{b} h^{2}$ is near or greater than this $\mathrm{BBN}$ value ( $\mathrm{HSa}$, and $\mathrm{Hu}$ \& Sugiyama 1996, hereafter HSc). To include its effects properly, we must solve the full equation (10). However, it is instructive to examine the qualitative origin of the effect first. Equation (6) tells us that the baryons contribute to the inertial and gravitational mass of the fluid. Thus, with a higher baryon content, gravity can compress the fluid more strongly inside the potential well $\Psi<0$. In the limit that $x \gg 1$ and we observe for only a time short compared with changes in $R$ and the source, the solution to the oscillator equation (6) is

$$
\Theta_{0}+\Psi=C_{1} \cos \left(k r_{s}\right)+C_{2} \sin \left(k r_{s}\right)-R \Psi .
$$

The last term represents the baryon drag on the photons and displaces the zero point of the oscillation. Since $-R \Psi>0$ inside a potential well, it enhances the compression and suppresses the rarefaction stage of the acoustic oscillation in the potential well. The crucial point is that the presence of baryons allows us to distinguish between the two stages from the observable rms deviation $\left|\Theta_{0}+\Psi\right|$. Figure 5 shows an example based on the source in equation (24) with $p_{1}=2, p_{2}=1$ and $\alpha=0.05$. Here we take the $k$-spectrum at recombination for a baryon content of $\Omega_{b} h^{2}=0,0.0125,0.25$, where $R_{*}=R\left(\eta_{*}\right)=0,0.38,7.6$. The neutrino and CDM densities have been set to zero for simplicity. Notice that if baryon drag is significant but not dominant (middle panel) then it will modulate the peaks into a pattern of alternating peak heights. If it dominates (bottom panel), the rms fluctuations no longer possess zero crossings but oscillate around some large d.c. offset. Either pattern is quite distinctive and, as we shall see, can separate the adiabatic from isocurvature scenarios in all cases save those with $|R \Psi| \ll\left|\Theta_{0}+\Psi\right|$ at last scattering.

\subsection{Photon Diffusion}

Finally, photon diffusion leaves a robust signature by providing a cutoff scale to the acoustic oscillations that is independent of the source fluctuation. As the diffusion length passes the wavelength, acoustic oscillations are exponentially damped (see Silk 1969; Weinberg 1972). Physically, this occurs since diffusion erases temperature differences across a wavelength and causes viscosity (or anisotropic stress) in the fluid. Anisotropic stress, or the quadrupole, is generated from the free streaming of a dipole fluctuation $\Theta_{1}$. As photons from crests and troughs of the 


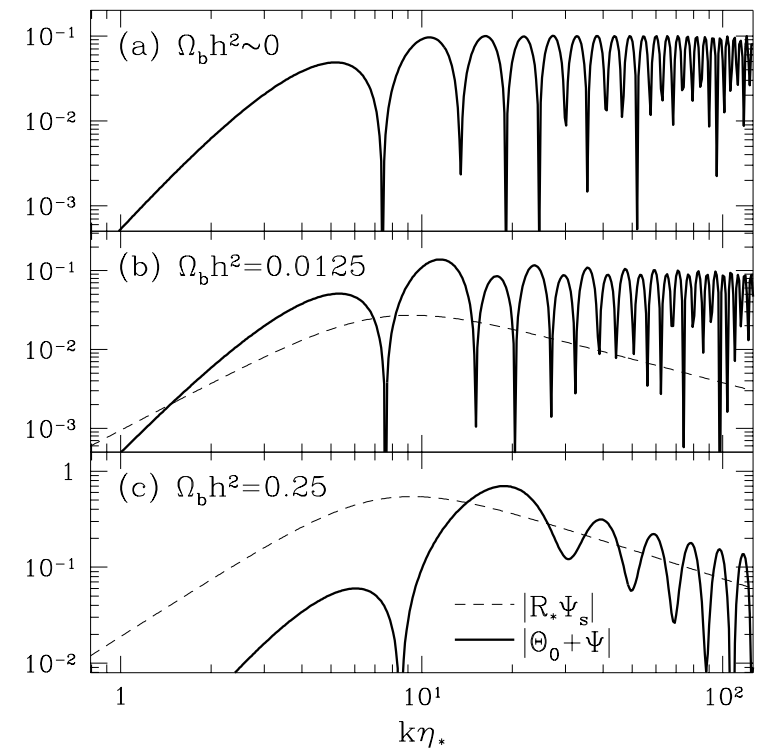

FIG. 5.-Baryon drag and relative peak heights. Baryons displace the zero point of the oscillation by $-R \Psi$. For a near BBN baryon content (center panel), the displacement is smaller than the oscillation itself leading to alternating peak heights in the rms. For a much larger baryon content, the photons oscillate around a strongly displaced zero point.

original velocity perturbation meet, their Doppler shifts create a quadrupole temperature pattern. This transfer of power from the dipole to the quadrupole is but a manifestation of a general tendency. Streaming transfers power to higher angular moments since the original temperature fluctuation subtends a smaller and smaller angle as seen by a distant observer. However, in the tight coupling limit, streaming is collisionally suppressed by the factor $\dot{\tau} / k$ (the optical depth through a wavelength) such that in the tight coupling limit, the anisotropic stress is approximately (see HSc, eq. [A8])

$$
\Pi_{\gamma}=\frac{8}{5}(k / \dot{\tau}) f_{2}^{-1} \Theta_{1},
$$

where $f_{2}=\frac{3}{4}$ (Kaiser 1983). Other approximations commonly employed are $f_{2}=9 / 10$ for unpolarized radiation (Chibisov 1972) and $f_{2}=1$ for further neglecting the angular dependence of Compton scattering (Weinberg 1972 ; HSa). From equation (26), it is natural to try a solution of the form $\exp i \int \omega d \eta$ for both $\left[\Theta_{0}+(1+R) \Psi\right]$ and $\Theta_{1}$. Heat conduction, proportional to $V_{b}-\Theta_{1}$, is described by iterating the baryon Euler equation (2) to second order,

$$
V_{b}-\Theta_{1}=-\dot{\tau}^{-1} R\left[i \omega \Theta_{1}-k \Psi\right]-\dot{\tau}^{-2} R^{2} \omega^{2} \Theta_{1} .
$$

Here we have ignored changes on the order of an expansion time compared with those at the oscillation period. Combining equations (27) and (28) in the photon Euler equation (2), we obtain the dispersion relation

$$
\omega= \pm k c_{s}+i \frac{1}{6} k^{2} \dot{\tau}^{-1}\left[\frac{R^{2}}{(1+R)^{2}}+\frac{4}{5} f_{2}^{-1} \frac{1}{1+R}\right]
$$

recalling that the sound speed $c_{s}=[3(1+R)]^{-1 / 2}$. It follows that acoustic oscillations are damped as (HSc)

$$
\Theta_{0}+\Psi=\left[\hat{\Theta}_{0}+\Psi\right] e^{-\left[k / k_{D}(\eta)\right]^{2}}+R \Psi\left(e^{-\left[k / k_{D}(\eta)\right]^{2}}-1\right),
$$

where $\hat{\Theta}_{0}$ is the acoustic signal described by equation (4) and the damping wavenumber is

$$
k_{D}^{-2}=\frac{1}{6} \int d \eta \frac{1}{\dot{\tau}} \frac{R^{2}+4 f_{2}^{-1}(1+R) / 5}{(1+R)^{2}} .
$$

To order of magnitude, the diffusion length is the geometric mean of the Compton mean free path $\dot{\tau}^{-1}$ and the horizon $\eta$, as expected of a random walk. Aside from the residual baryon drag effect, photon diffusion leaves a distinctive damping signal in the CMB that is only dependent on the cosmological background. Because the damping is exponential, no oscillations survive at $k \gg k_{D}$ regardless of the source.

One complication arises however. As recombination progresses, the ionization fraction and hence the differential optical depth $\dot{\tau}$ decreases. The corresponding increase in the damping length can still be approximated in the tight coupling limit if $\dot{\tau} / k_{D}\left(\eta_{*}\right) \sim k_{D} \eta_{*} \gg 1$. In this case, the diffusion length passes the wavelength while tight coupling holds and the damping can be calculated semianalytically from the known ionization history (see HSc). If this condition is not satisfied, as is the case for extremely low $\Omega_{b} h^{2}$ or reionized models with a long Compton mean free path at recombination, the damping must be calibrated numerically ( $\mathrm{Hu} \&$ White, in preparation).

\subsection{Summary}

In summary, then, we have shown that the evolution of fluctuations acts to resist changes in the spatial curvature whenever the pressure can be neglected (outside the horizon). Schematically, this is because the radiation or matter in a growing curvature perturbation redshifts faster than average (cf. cosmological redshift, as in $\S 2.1$ ) reducing the local energy density and hence stabilizing the curvature perturbation. It is also a consequence of causality as shown in the Appendix.

The behavior of the acoustic oscillations due to sources outside the horizon thus depends only on the initial conditions: whether or not there are initial uncompensated curvature perturbations. The compensation by the radiation also implies that the self-gravity of the photons produces an important feedback mechanism. The result is that sources arising before or near horizon crossing stimulate either the cosine or the sine mode of the oscillation, not an arbitrary admixture.

The fact that the compensation mechanism leads to rarefactions in the photon density at horizon crossing for isocurvature fluctuations but compressions for adiabatic fluctuations inside the potential well is an extremely robust conclusion. Of crucial importance is that it leads to an observable effect in models with $\Omega_{b} h^{2}$ of a few percent or greater due to the drag induced by the baryons. Photon diffusion also leaves a distinct damping tail that is entirely independent of the model for the fluctuations but sensitive to the thermal history.

\section{ACOUSTIC SIGNATURES}

Having studied the acoustic oscillations in the last section, let us now consider what signatures the effects summarized in $\S 2.9$ leave in the angular power spectrum of CMB anisotropies. Acoustic oscillations are frozen in at last scattering $\eta_{*}$ when the Compton optical depth to the present drops below unity. The evolutionary properties of 
the acoustic phenomena before last scattering influence the peak locations, heights, and damping tail. These will be used in the next sections to devise tests of the model for structure formation and the background cosmology.

\subsection{Peak Locations for Driven Oscillations}

At last scattering, each $k$-mode is caught at a different phase in its oscillation. Of course, one can adjust the magnitudes in $k$ by choosing different relative weights for the $k$-modes at the initial conditions. We assume that any such weighting is not in itself oscillatory in $k$. Under this single assumption, the acoustic pattern of peaks is robust to the details of the model even though their absolute heights are not. These oscillations on the last scattering surface are viewed today as anisotropies on the sky. Thus, the $k$-space power spectrum is projected onto an angular power spectrum. The angular correlation function is broken up into $l$ Legendre moments, which represent the power on the scale $\theta \sim l^{-1}$. The power spectrum is usually denoted as $l(l+1) C_{l}$, where $C_{l}$ is the ensemble average of the squared multipole coefficients.

As we shall see in $\S 5$ (see eq. [39]), since the projection of $k$ onto $l$ is highly dependent on the curvature, knowledge of the physical scale of acoustic features allows a sensitive probe of the curvature. The physical scale of the peaks is related to the sound horizon $r_{s}=\int c_{s} d \eta \approx \eta / 3^{1 / 2}$ as $R \rightarrow 0$, or more generally,

$$
r_{s}=\frac{2}{\sqrt{3}}\left(\Omega_{0} H_{0}^{2}\right)^{-1 / 2} \sqrt{\frac{a_{\mathrm{eq}}}{R_{\mathrm{eq}}}} \ln \frac{\sqrt{1+R}+\sqrt{R+R_{\mathrm{eq}}}}{1+\sqrt{R_{\mathrm{eq}}}},
$$

assuming the universe was radiation dominated before $a_{\text {eq }}$. The exact relation of the peak scale to the sound horizon depends on the nature of the fluctuations and supplies a test of the model (see $\S 5.6$ ). Let us now examine this relationship more carefully.

Once the source becomes ineffective $\left|\Theta_{0}\right| \gg\left|\Phi_{s}\right|$, the partition into pure mode amplitudes $\mathscr{A}_{a}$ and $\mathscr{A}_{b}$ of $\S 2.4$ becomes time independent and the acoustic oscillations settle into a pure sinusoidal form, $\sin \phi(\eta, k)$, with phase

$$
\phi=k r_{s}+\tan ^{-1}\left(\mathscr{A}_{a} / \mathscr{A}_{b}\right) \text {. }
$$

For sources that peak before horizon crossing, backreaction effects create a two-state system (see $\S 2.5$ ). If the fluctuations are not perfectly compensated at the initial conditions, $\mathscr{A}_{a} \gg \mathscr{A}_{b}$. Compensation tends to create a situation where $\mathscr{A}_{b}>\mathscr{A}_{a}$.

Robust predictions arise for the location of the peaks in the $k$ or $l$ space power spectra. Since the phase is dependent only on the nature of the initial fluctuation, not on the detailed behavior or the source in time or $k$, all such models give definite predictions for the peak locations:

$$
\phi\left(k_{m}\right)=(m-1 / 2) \pi,
$$

for integer $m$. Furthermore, the harmonic series of peak locations is independent of the background quantities that fix the sound horizon and the angle it subtends on the sky. The generic adiabatic prediction is that peak locations follow a $1: 2: 3 \ldots$ series in $k$ or $l$. Isocurvature models tend toward a $1: 3: 5 \ldots$ pattern (HSb). Even if $\mathscr{A}_{a}$ and $\mathscr{A}_{b}$ are comparable, as would be the case if a small (finely tuned) uncompensated fluctuation remained in the initial condi- tion, the pattern of peaks uniquely determines the phase shift. This might be the case if the initial conditions contain a balanced amount of coherent adiabatic and isocurvature fluctuations. In most physical examples, however (see, e.g., Kawasaki, Sugiyama, \& Yanagida 1995), the processes that generate the two types of fluctuations are statistically independent and the two contributions are incoherent, i.e., generated with different phases in $k$. In this case it is even more unlikely that the two contributions would be balanced to give the same acoustic amplitudes. For incoherent superpositions, the phase is always determined by the dominant component with a change in amplitude, not phase, caused by the weaker component. Furthermore, the phase difference $\phi\left(k_{m}\right)-\phi\left(k_{m-1}\right)=\pi$ implies that regardless of the phase shift or amplitude variation, the spacing of the peaks in $k$ is given by

$$
k_{A}=k_{m}-k_{m-1}=\pi / r_{s} .
$$

Thus, the acoustic peaks possess both model-dependent information in the ratios of their locations and modelindependent information for the measurement of background parameters in the spacing between the peaks.

\subsection{Peak Locations for Forced Oscillations}

Although definiteness in phase is a typical feature of acoustic oscillations, it is not necessarily obeyed by models in which the source is still active inside the horizon. In this case, the partition into pure modes through $\mathscr{A}_{a}$ and $\mathscr{A}_{b}$ is time dependent. There are two limiting cases worth considering. The source could be scale-free in its temporal behavior such that $\Phi_{s}(x, k)=G(x) F(k)$, where recall $x=k \eta$. This occurs, for example, if the source behavior is correlated with horizon crossing perhaps due to the onset of a causal mechanism. As discussed in $\S 2.6$, this is in fact the most likely scenario since metric perturbations that can overcome photon pressure become increasingly hard to generate inside the horizon. In this case, the behavior as a function of $x=k \eta$ represents both the time evolution of a fixed $k$-mode and the transfer function in $k$ for a fixed time, e.g., at last scattering $\eta_{*}$. If $G(x)$ peaks around $x=1$ as is typical, we would expect an irregular first peak followed by an increasingly regular but phase-shifted harmonic series. If it peaks much after $x=1$, complicated acoustic behavior containing detailed information about the source evolution would result (see Fig. 4). Even in this case, we can see from equation (25) that if the source is slowly varying in time such that $G^{\prime \prime \prime \prime} \ll c_{s}^{2} G^{\prime \prime}$, the natural period is still imprinted in the oscillations. In cases where the driving force is large, we expect only compressional phases to be clearly visible as peaks in the rms. Rarefactions become rms troughs. Using only the compressional extrema, the peak spacing is then given by $2 k_{A}=2 \pi / r_{s}$

In the extreme case that the metric perturbations are generated well after $x=1$, one might also expect stochastic behavior in the source (Albrecht et al. 1996). Here the timing mechanism due to the act of horizon crossing no longer serves to correlate the modes. In this case, each $k$-mode receives a different set of impulses from the source. The phase shift given by $\tan ^{-1}\left[\mathscr{A}_{a}(x, k) / \mathscr{A}_{b}(x, k)\right]$ varies with $k$. In the extreme limit of rapid variation, the phase information is lost when summing over $k$-modes to form the observable anisotropy. This washes out the oscillatory behavior. It is believed that a concrete example of this mechanism is given by the cosmic string scenario (Albrecht 
et al. 1996) although whether it is the dominant mechanism or not is currently disputed. We shall discuss what can be learned in the case where the oscillations are washed out in $\S 5$. Let us assume for the present that oscillations will be observed.

\subsection{Peak Heights}

A wealth of information is stored in the peak heights. Their signature is more model dependent than the peak locations and provides an excellent means of examining the fine details of the model. On the other hand, robust features that distinguish between general classes of models are more difficult to isolate. In this section, we will examine generic features in the peak heights that may at least be used as clues for this purpose.

Let us begin by examining the familiar scale-invariant adiabatic case. In the tight coupling limit, there are two effects that determine the heights of the peaks: the driving force of feedback and baryon drag. They are to a certain extent mutually exclusive. As we have seen in $\S 2.5$, feedback boosts the oscillation amplitude above the initial conditions $\left[\Theta_{0}+\Psi\right]\left(\eta_{i}, k\right)=\frac{1}{2} \Psi\left(\eta_{i}, k\right)$ by a factor of 3 . Recall that the fluid first is compressed by its own self-gravity. Photon pressure resists the compression, causing the photonbaryon contribution to the potential to decay. The fluid is thus released exactly in this highly compressed state into the acoustic phase. We show in the Appendix that the potential in the matter-dominated limit $\Psi(\eta, k)=(9 / 10) \Psi\left(\eta_{i}, k\right)$, thus the boost represents a factor of 5 enhancement over the $\Theta_{0}+\Psi=\frac{1}{3} \Psi$ Sachs-Wolfe tail. The inclusion of the neutrino background slightly lowers the amplitude (see HSc). The driving effect does not occur if the potentials are dominated by an external source such as cold dark matter. Hence, the prominence of the acoustic oscillations increases if the universe is made more photon-baryon or radiation dominated at last scattering.

Baryon drag enhances the compressional, here odd, peaks by a term of $\mathcal{O}(R \Psi)$. This alters the peak heights to give the distinctive alternating or offset oscillation pattern but becomes subdominant if the potential is intrinsically small. Such is the case for driven oscillations since the potential $\Psi \approx \Psi_{\gamma b}$ and decays inside the sound horizon.

For isocurvature models, gravitational redshifts cause the effective temperature above the horizon to be $\Theta_{0}+\Psi \approx$ $2 \Psi<0$ (see $\S 2.2$ ). It is also worthwhile to note that although the fluctuation outside the horizon at last scattering is small in these models, this does not imply that the observable anisotropy from those scales is correspondingly small. Gravitational redshifts from the time-dependent potential continue to generate fluctuations between last scattering and the present in the same manner. This is generally called the integrated Sachs-Wolfe (ISW) effect. Thus, an isocurvature model that generates a scale-invariant spectrum of curvature fluctuations near horizon crossing yields a flat large-scale anisotropy spectrum just as in the familiar adiabatic case (Bennett, Stebbins, \& Bouchet 1992; Coulson et al. 1994; Pen, Spergel, \& Turok 1994).

Superhorizon isocurvature evolution makes the photonbaryon fluid more and more rarefied inside a potential well until photon pressure can successfully resist rarefication. Since the first feature is the turning point from the superhorizon behavior, it is not prominent in comparison to the $2 \Psi$ Sachs-Wolfe effect. However, the fluid then begins infall into the source wells. The driving effect of the photon- baryon contribution to the potential wells now proceeds as in the adiabatic case to enhance the oscillation, making the second feature much more prominent than the first (see Fig. $3)$. Whereas the first peak has a height of order $2 \Psi$ at $k r_{s}=$ $\pi / 2$ and is small since compensation eliminates metric fluctuations above the horizon, the second peak has a height of order $2 \Psi$ at $k r_{s}=3 \pi / 2$, which is significantly larger since the photon-baryon contribution adds to rather than cancels the source. As in the adiabatic case, baryon drag contributes an $\mathcal{O}(R \Psi)$ term that boosts the compressional phases. However, in the isocurvature case, these are the even peaks since compensation demands that if the first feature occurs near the horizon it represents rarefaction inside the potential well. Thus, the second isocurvature peak is lifted even higher with respect to the first by baryon drag.

How robust are these general tendencies? The prominence of the acoustic oscillations compared with the largescale tail can be masked or altered by the presence of tilt or features in the initial $k$-spectrum as well as by other effects from tensor and vector metric perturbations (e.g., Crittenden \& Turok 1995). These effects are, however, unlikely to obscure the distinctive alternating peak heights due to baryon drag. Unfortunately, the baryon drag effect may be difficult to observe if $|R \Psi|\left(\eta_{*}\right) \ll\left|\Theta_{0}+\Psi\right|\left(\eta_{*}\right) \sim$ $|\Psi|\left(\eta \approx 1 / c_{s} k\right)$. Even with high precision measurements, one must first remove the diffusion damping envelope in this case.

\subsection{Damping Tail}

Unless baryon drag dominates over the acoustic oscillation, as in the case that $|R \Psi|\left(\eta_{*}\right) \gg\left|\Theta_{0}+\Psi\right|\left(\eta_{*}\right)$, the damping leaves a clear signal in the CMB. To demonstrate this robustness to changes in the model for the gravitational source, we compare in Figure 6 the anisotropy power spectra of a standard inflationary model with scaleinvariant curvature fluctuations at horizon crossing and a similar axionic isocurvature model. The background parameters are set to be equal at $\Omega_{0}=1.0, h=0.5$ and

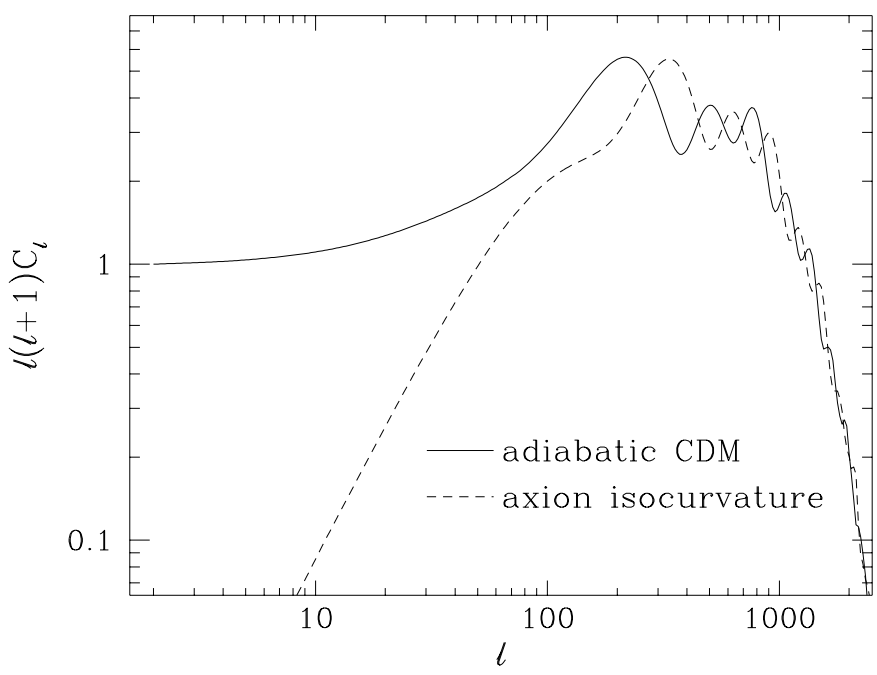

FIG. 6.-Diffusion damping. Although adiabatic and isocurvature models predict acoustic oscillations in different positions, they both suffer diffusion damping in the same way. The damping length is fixed by background assumptions, here $\Omega_{0}=1, h=0.5, \Omega_{b}=0.05$ and standard recombination. These calculations were performed using a full numerical integration of the Boltzmann equation with the code of Sugiyama (1995) as were results in Figs. 7, 8, 10, and 11 . 
$\Omega_{b}=0.05$. Notice that the damping behavior is independent of the nature of the fluctuations.

The angular location of the damping tail is highly sensitive not only to the curvature, which dominates projection effects (see $\S 5$ ), but also to the thermal history, which sets the maximum diffusion scale, the baryon content $\Omega_{b} h^{2}$, which sets the mean free path of the photons, and the matter content $\Omega_{0} h^{2}$, which sets the horizon scale. A measurement of the damping scale alone would fix the combination of these quantities defined by equation (31). More specifically, if last scattering occurs due to standard recombination, the wavelength $k_{D}$ at which the acoustic amplitude falls to $e^{-1}$ of its original amplitude can be fitted by semianalytic techniques (HSc, eq. [E6]) to $\sim 10 \%$. Unfortunately, a measurement of $k_{D}$ cannot break the degeneracy in these quantities. If $\Omega_{b} h^{2}$ is constrained by BBN and $\Omega_{0} h^{2}$ by dynamical mass and Hubble constant measurements, it would provide interesting constraints on the curvature of the universe as we shall show in $\S 5.3$. Furthermore, with a measurement of the acoustic peaks themselves, the degeneracy can potentially be removed in many models for the source fluctuations (see $\S 5.6$ ).

\section{UNIQUENESS OF THE INFLATIONARY SPECTRUM}

The inflationary paradigm is the front running candidate for a mechanism of fluctuation generation in the early universe: the perturbations in density that are the precursors of galaxies and $\mathrm{CMB}$ anisotropies today. Are there unique signatures of inflation that can validate the paradigm? For a long time it was thought that a nearly scale-invariant spectrum of anisotropies would provide evidence for inflation (Kolb \& Turner 1990). But then it was realized that models of structure formation based on topological defects also naturally formed scale-invariant spectra (see $\S 3.3$ and Bennett et al. 1992; Coulson et al. 1994; Pen et al. 1994). From the other direction, finding that the universe had nonvanishing spatial curvature once seemed like a way to "disprove" inflation, however recently it has been shown that inflation could survive such a revelation (Bucher, Goldhaber, \& Turok 1994; Yamamoto, Sasaki, \& Tanaka 1995).

Currently, the most popular means of "proving" inflation is to test the consistency relation between the tensor (gravity wave) and scalar (density) modes. By measuring the detailed shape of the anisotropy spectrum, one may infer the relative amplitudes of scalar and tensor perturbations. The ratio of tensor to scalar contributions is proportional in slow-roll inflation to the slope of the tensor spectrum (Liddle \& Lyth 1993; Davis et al. 1992; Turner \& White 1996). However, this method of proof requires a precise determination of the tensor to scalar ratio, which in turn requires a sizable fraction of the anisotropy be contributed from tensor modes (White, Krauss, \& Silk 1995; Knox \& Turner 1994). What one really desires is a test based on the most basic ideas of the inflationary scenario that will be both observationally feasible and able to survive the ingenuity of model builders.

The key feature of inflation for our purposes is that it provides a mechanism of connecting parts of the universe at early times, which are currently spacelike separated, i.e., outside the horizon. In fact, it can be shown that inflation is the unique causal mechanism for correlating curvature perturbations on scales larger than the horizon (Liddle 1995; $\mathrm{Hu}$, Turner, \& Weinberg 1994). The possibility of a white noise spectrum of superhorizon curvature perturbations is discussed in the Appendix but is already observationally ruled out as a means of structure formation. The question therefore arises, are there unique consequences of such superhorizon curvature perturbations? If so, their observation would provide a "proof" of the inflationary paradigm.

\subsection{Robustness of the Harmonic Series}

We have seen in $\S 3.1$ that the harmonic series of acoustic peaks in a model with superhorizon curvature fluctuations is given by the cosine series $1: 2: 3 \ldots$ for the locations of the peaks. Compensated superhorizon fluctuations follow the sine series $1: 3: 5 \ldots$ Thus, by measuring the ratio of the first three peak positions in $l$ space, these two possibilities may be distinguished. There are two concerns that need to be addressed for this potential test of inflation. How robust is the harmonic prediction in the general class of inflationary models? Can an isocurvature scenario in which fluctuations are generated inside the horizon mimic an inflationary series?

Let us consider the first question. Residual driving effects can distort the shapes and locations of the first few peaks. Even for the pure adiabatic mode of $\S 2.4$, the peaks have not completely settled into their asymptotic forms until $k r_{s} \gg 1$ and the peak positions follow the series in $\Theta_{0}+\Psi$,

$$
0.88: 1.89: 2.93: 3.94: 4.96 \ldots
$$

We will hereafter refer to these ratios as the "cosine series," despite the fact that it has not yet converged upon an actual cosine oscillation by the first few peaks. In particular, the first peak is somewhat low in $l$ compared with the expectation from the higher peaks. Let us compare this prediction against actual inflationary models from a numerical calculation (see Fig. 7, left panel, solid lines). All models do indeed exhibit the harmonic structure predicted by equation (36).

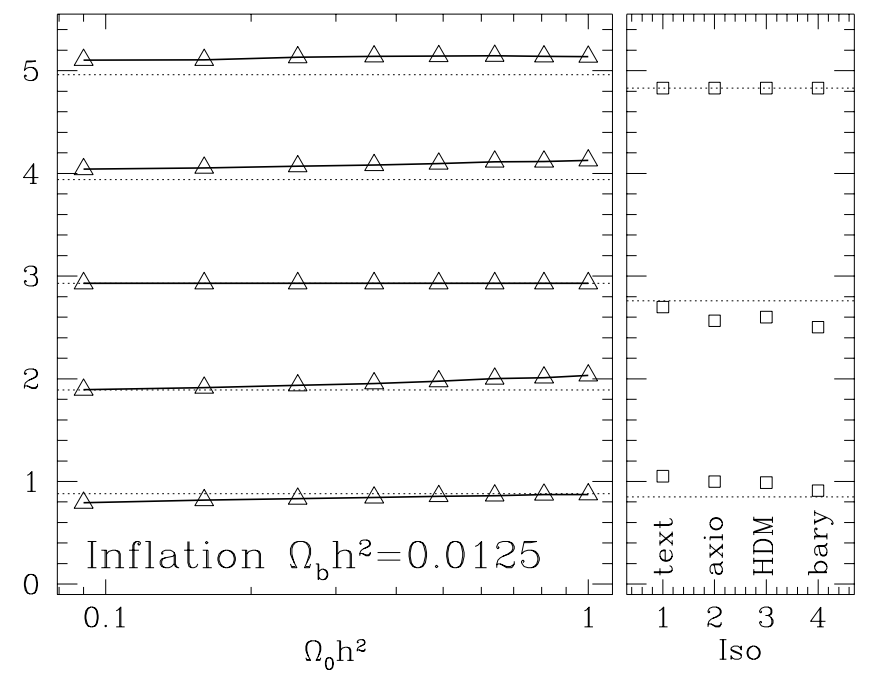

FIG. 7.- Relative peak locations and the harmonic series. In the ideal case, inflationary acoustic oscillations follow the cosine series and driven isocurvature models a sine series (light dotted lines). The ISW effect, baryon drag and diffusion damping serve to distort the peak locations. The isocurvature cases considered are baryon isocurvature (HSb); textures (Crittenden \& Turok 1995); axionic isocurvature (Kawasaki et al. 1995); and hot dark matter isocurvature (de Laix \& Scherrer 1995). Numerical calculations (points) are normalized to the ideal predictions of eqs. (37) and (38) at the third peak and are specifically for $\Omega_{0}+\Omega_{\Lambda}=1$, though this constraint is irrelevant for the peak rations. They demonstrate that the two cases remain quite distinct especially in the ratio between the first and third peak. 
There is a slight shift of the first few peaks downward in $l$ as $\Omega_{0} h^{2}$ is lowered. As noted by $\mathrm{HSb}$, if the radiation is still dynamically important after last scattering, the resultant ISW effect will shift power toward larger angles and distort the first peak. The even peaks are also shifted upward as $\Omega_{0} h^{2}$ increases to make potentials deeper and baryon drag more important. Still, for reasonable matter content and a near BBN baryon content a ratio of third to first peak of $l_{3} / l_{1} \approx 3.3-3.7$ is a robust prediction of inflation. Furthermore, the ratio of the first peak location to the peak spacing is between $l_{1} / l_{A} \approx 0.7-0.9$. The peak spacing is best measured by the prominent peaks, e.g., for inflation $l_{A}=$ $\left(l_{3}-l_{1}\right) / 2$. The only caveat is that models with extremely high $\Omega_{b} h^{2} \gtrsim 0.04$ and $\Omega_{0} \gg \Omega_{b}$ may be dominated by the baryon drag effect. In this case, only the compression phases are visible as peaks in the rms. Thus, every other oscillation is unobservable and the series becomes $1: 3: 5 \ldots$ like the isocurvature spectrum. For a model with reasonable matter content $\Omega_{0} h^{2} \lesssim 0.25$, no value of $\Omega_{b} h^{2}$ entirely eliminates the second peak. For $\Omega_{0} h^{2}=0.64$, the second peak disappears for $0.04 \lesssim \Omega_{b} h^{2} \lesssim 0.5$ and the fourth for $0.128 \lesssim$ $\Omega_{b} h^{2} \lesssim 0.32$. Even in this extreme case, only a gross violation of BBN would entirely mask the inflationary pattern. Thus, the harmonic series is a robust prediction of inflation.

\subsection{Uniqueness of the Harmonic Series}

Is the cosine harmonic series a unique prediction of inflation? Just as in the inflationary case, for isocurvature models residual driving effects create a downward shift from the $1: 3: 5 \ldots$ pattern. Even a pure driven isocurvature model with a source $\Phi_{s} \propto x^{-1}$, which converges to the sine series for $k r_{s} \gg 1$ (see $\left.\S 2.5\right)$, gives

$$
0.85: 2.76: 4.83: 6.89: 8.91 \ldots
$$

We will hereafter refer to these ratios as the "sine series." How closely do real models follow this prediction? For comparison, we show in the right panel of Figure 7 the ratios of the peak locations for common isocurvature models found in the literature: the baryon isocurvature (HSb), textures (Crittenden \& Turok 1995), axionic isocurvature (Kawasaki et al. 1995), and HDM isocurvature (de Laix \& Scherrer 1995). Notice that these models roughly correspond to the sine series prediction. Thus, in all inflationary models with reasonable baryon content, the ratio of peak locations should be distinguishable from the current models based on the isocurvature restriction. This suggests that to mimic inflation an isocurvature model would have to either possess extreme conditions or fine tuning.

Let us attempt to construct models that mimic inflation. Since the first inflationary peak appears immediately after sound horizon crossing $k=\pi / r_{s}$, it is difficult to avoid or counter the driving effects that produce the sine series. Can a series based on peaks arising after sound horizon crossing somehow mimic the cosine series? Consider the possibility that the first isocurvature peak in the sine series equation (37) is missing or cannot be observed. This is indeed likely in some models since the first peak can be quite low in intrinsic amplitude (see Fig. 8, $\S \S 3.3$ and 4.3). Furthermore, external effects such as tensor and vector modes that naturally possess a feature on the horizon scale at last scattering might mask or distort the peak. In this case, the observable peaks would follow the series: $2.8: 4.8: 6.9 \ldots$, which is close enough to the cosine series to cause some concern, especially if only the first two peaks are measured. More gener-

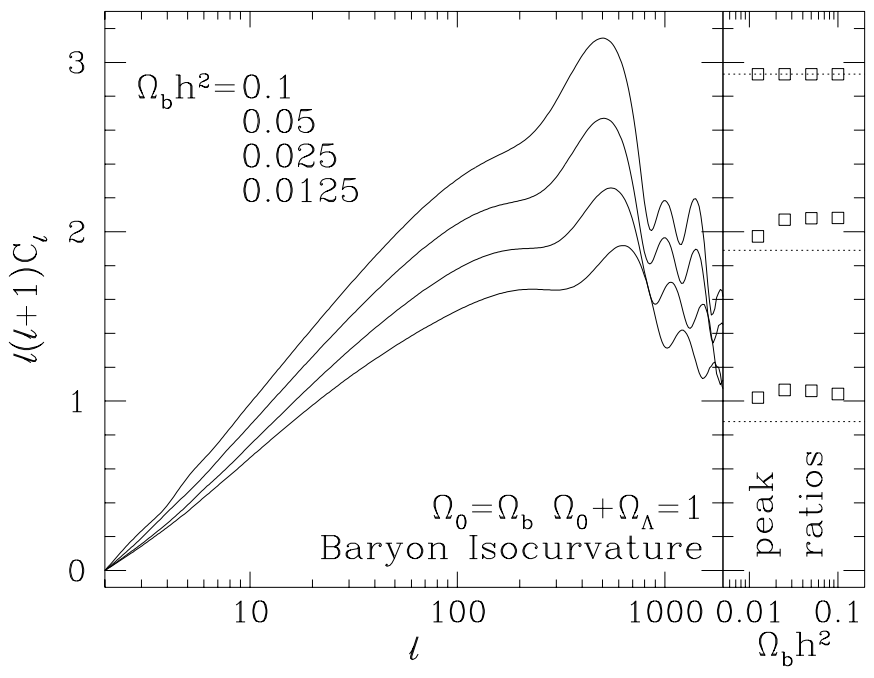

FIG. 8.-Obscured isocurvature peak. The first isocurvature "peak" appears as a shoulder and may be obscured especially in high baryon models where the second peak is significantly higher (left panel, arbitrary normalization). If the second peak starts the harmonic series, the ratios (right panel, points) can be quite close to the cosine prediction (dotted lines) The points are normalized to the cosine prediction at the third peak. These models can be distinguished by the peak to spacing ratio and the morphology of the first compressional peak (second feature).

ally, one might have a source that turns on only after sound horizon crossing so that the harmonic series is again shifted toward smaller scales. These possibilities, however, create spectra that are not as close to the inflationary prediction as they initially might appear.

The crucial point is that although the starting point of the harmonic series can change with the model, its spacing cannot. The separation between the peaks is fixed by the sound horizon at last scattering. Thus, the harmonics cannot simply be scaled to match the inflationary prediction. The distinction is clearer when we consider the ratio of the peak location to peak separation. The idealized inflationary prediction of equation (36) requires a ratio of the first peak to the separation between peaks of $l_{1} / l_{A} \approx 0.88$. If the second peak of the isocurvature prediction equation (37) is to be taken for a first peak, it gives a corresponding ratio of $l_{1} / l_{A} \approx 1.33$. In other words, we expect a factor of 1.5 difference between the two cases. The uncertainty displayed in the real models of Figure 7 do not destroy the test. When comparing models with the same values for $\Omega_{0} h^{2}$ the idealized expectation is validated, e.g., between inflation and axionic isocurvature with $\Omega_{0} h^{2}=0.25$ and $\Omega_{b} h^{2}=0.0125$ there is a $1.4-1.5$ difference in $l_{1} / l_{A}$ depending on which peaks are used to measure the spacing. Even if the background parameters are unknown, to get the ratio as low as it is in the highest inflationary case $\left(l_{1} / l_{A} \approx 0.9\right.$ for $\Omega_{0} h^{2} \lesssim$ 1 ), the first peak must be created around sound horizon crossing.

The robustness of this test arises because the natural frequency of the oscillator is related to the sound horizon. Thus, the oscillations, if regular enough to be mistaken for inflation, must appear regularly spaced with respect to that scale. It is of course in principle possible that the external source is periodic and drives the oscillator at a different frequency. It is amusing to note that even this rather unlikely scenario can be distinguished. The damping tail provides a scale that is entirely independent on the nature of 
the fluctuations. If the background parameters are known, the ratio of the damping scale to peak position provides an additional consistency test for the inflationary scenario. In models where the fluctuation is generated inside the sound horizon, the ratio $l_{1} / l_{D}$ increases. In particular, for the above case of a missing first isocurvature peak, the ratio increases by a factor of 1.5 exactly as with the case of the peak-tospacing ratio.

Therefore, the only way to mimic the inflationary series is to tune the behavior of the source at sound horizon crossing so that it immediately generates a peak in the proper position but leaves no residual effects that would distort the series of higher peaks. Although contrived, this is possible. In Figure 9, we plot a source given by equation (24) with $p_{1}=2, p_{2}=1$, and $\alpha=0.05$ with $\Omega_{b} \rightarrow 0$. In this case, we have constructed a source that dies off after $x \approx \alpha^{-1}$ such as to leave the oscillations in a nearly pure cosine mode. The first five peaks follow a series $1: 2.05: 3.16: 4.23: 5.29$, very close to the canonical inflationary prediction. Notice, however, that the prediction is $180^{\circ}$ out of phase with the inflationary prediction. This is an important fact that we will make use of in the next two sections. As we shall see, the crucial point is the effect of compensation near sound horizon crossing. One should also bear in mind that we have tuned the source to produce as pathological a case as possible. The cosine harmonic of peaks is essentially but not entirely unique to the inflationary paradigm. Its confirmation would strongly support the inflationary scenario.

\subsection{First Peak}

Can we employ additional information to eliminate the possibility that an isocurvature model might mimic the inflationary model through the peak positions? Compensation from photon feedback discussed in $\$ \S 2.4$ and 3.3 provides an essential distinction. Near or above the horizon, the photons act to resist any change in curvature produced by the source if the universe is radiation dominated (see Appendix for relaxations of this assumption). Thus, the first peak in an isocurvature model, if it is sufficiently close to the horizon to be confused with the infla-

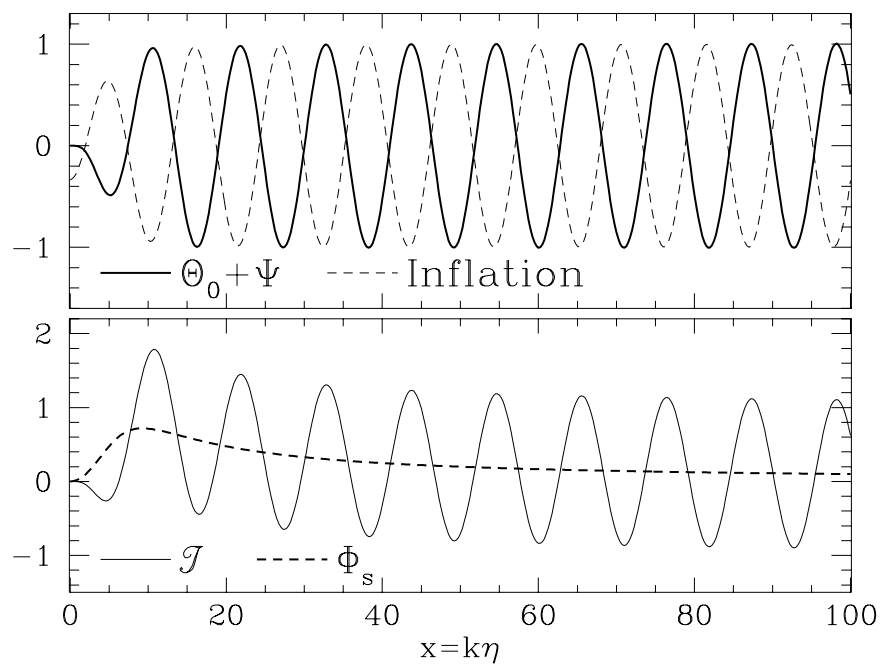

FIG. 9.-Pathological isocurvature model. Here forced isocurvature fluctuations are tuned to match the locations of the inflationary prediction (upper panel, dotted lines) with vanishing baryon content $R \rightarrow 0$. Notice that even in this case the isocurvature oscillations are out of phase with the inflationary prediction by $180^{\circ}$. With the inclusion of baryon drag, this leaves an observable signal in the rms. tionary prediction, must be anticorrelated with the source. In other words, the first peak in the rms temperature represents the rarefaction stage inside the potential well of the source rather than a compression phase as in the inflationary prediction.

Since the dilation effect $\dot{\Theta}_{0}=-\dot{\Phi}$, which causes $\Theta_{0}+\Psi \approx-2 \Phi$, creates both the first peak and the SachsWolfe effect, the first feature in an isocurvature spectrum is not truly a peak but a smooth turnover from the large-scale behavior as discussed in $\S \S 2.5$ and 3.3. This point represents the epoch at which the fluctuation ceases to follow the growth of the curvature and turns around to start the acoustic oscillation. It creates the shoulder appearance of the first isocurvature feature (see, e.g., Figures 6 and 8). On the other hand, the inflationary fluctuation in the effective temperature is proportional to cos $\left(k r_{s}\right)$ and passes through a zero at $k r_{s}=\pi / 2$ before the first peak (see Fig. 3). The resultant spectrum thus exhibits a rather sharp break between the gravitationally dominated Sachs-Wolfe tail and the first peak (see Fig. 6). The gravitational nature of the first peak in an isocurvature model makes it substantially less prominent when compared with the low $l$ tail. The first isocurvature peak also tends to be low with respect to the higher peaks because it appears too close to horizon crossing to experience the full forcing effect by the source.

Unfortunately, these morphological distinctions are hard to quantify. Indeed, the sharp rise to the first peak in the inflationary model can be masked by the presence of the integrated Sachs-Wolfe effect in a low $\Omega_{0} h^{2}$ universe (HSa). In an isocurvature model, the prominence of the first feature can be enhanced by a smooth bend in the power spectrum or vector and tensor modes. Nevertheless, the physical distinction between the first adiabatic and isocurvature peaks does suggest a robust way of distinguishing the models, as we shall now discuss.

\subsection{Relative Peak Heights}

Whereas the inflationary spectrum obeys a compressionrarefaction-compression pattern an isocurvature model displays a rarefaction-compression-rarefaction pattern. In the absence of baryons, there is no observable distinction between compression and rarefaction since only the rms can be measured. However, as we have seen in $\S 2.7$, baryons enhance the compression at the expense of rarefaction leading to an alternating series of peaks in the rms. In fact, the example in Figure 5 is the same pathological model of $\S 4.2$, which has peaks at the inflationary locations by construction. Notice that for reasonable baryon content, the even peaks are enhanced by the baryons, whereas the odd peaks are enhanced under the inflationary paradigm. Since this nonmonotonic peak pattern cannot be reproduced without introducing the appropriate features in the initial $k$ spectrum of fluctuations, the pattern of anomalously high odd cosine peaks is a unique feature of standard inflationary models.

Of course, an isocurvature model can also begin with a compression if the first peak is so low in amplitude as to be unobservable (see Fig. 8). However, such a model cannot simultaneously create the peak positions of the inflationary prediction. In fact, regardless of the peak positions, isocurvature models are still unlikely to mimic a strongly alternating heights pattern. Since the first compressional isocurvature peak occurs well after horizon crossing, neither the potentials of the source nor the photon-baryon 
backreaction are large enough to cause significant baryon drag unless the baryon fraction is exceedingly high. In this case, the behavior of the potential implies an anomalously high first compressional peak compared with the second due to baryon drag from the residual self-gravity (see Fig. 8).

Although this pattern of peak locations and relative heights is unique, it is not an entirely robust prediction of inflation. The intrinsic fractional effect at the $m$ th peak is on the order

$$
R\left(\eta_{*}\right) \frac{\Psi\left(\eta_{*}, m k_{A}\right)}{\Psi\left(\eta_{i}, m k_{A}\right)} \approx R\left(\eta_{*}\right) T\left(m k_{A}\right) \quad\left(\eta_{*} \gtrsim \eta_{\mathrm{eq}}\right)
$$

where $T(k)$ is the matter transfer function (Bardeen et al. 1987). However, if the intrinsic effect is small, complications such as possible smooth tilts in the initial spectrum and diffusion damping will make the signal difficult to observe (see Fig. 10). For $\Omega_{0} h^{2} \approx 0.25$ this occurs if $\Omega_{b} h^{2} \lesssim 0.007$ and for BBN baryon content for $\Omega_{0} h^{2} \lesssim 0.1$. Still, with high precision experiments, a sufficiently smooth initial spectrum, and known thermal history, it is possible that even these extreme cases may be testable $(\mathrm{Hu} \&$ White, in preparation). On the other hand if $R\left(\eta_{*}\right) T\left(m k_{A}\right) \gg 1$, baryon drag dominates and the even $m$ peaks (rarefactions) no longer appear as peaks in the rms (see Fig. 5). In this case, the oscillations are small compared with the offset and the distinction between compression and rarefaction stage is that they are maxima and minima of the rms, respectively. Since $T(k) \leq T(0)$, a necessary but not sufficient condition for this to occur is $R>1$ or a baryon content more than 3 times the BBN value.

\subsection{Summary}

In summary, inflationary models carry an acoustic signature with distinct properties that can distinguish them from isocurvature models. The ratio of peak locations is a robust prediction of inflation. In particular, the ratio of third to first peak location should be in the range $l_{3} / l_{1} \approx 3.3-3.7$ and the ratio of first peak location to peak spacing should be between $l_{1} / l_{A} \approx 0.7-0.9$. If this pattern is not observed in the $\mathrm{CMB}$, either inflation does not provide the main source of perturbations in the early universe or BBN grossly

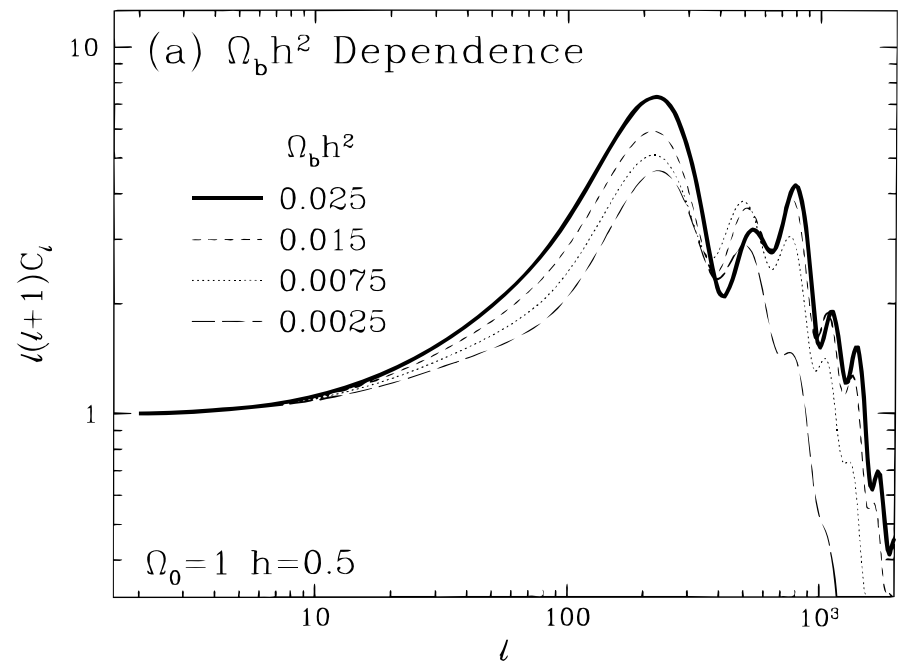

underestimates the baryon fraction. The latter possibility is treated more fully in $\S 5.6$. Observational confirmation of the pattern would provide a plausibility proof for inflation. It would thus require fine tuning for an isocurvature model to reproduce this spectrum. To close this loophole in the test of inflation, the relative peak heights can be observed. Assuming the location of the peaks follows the inflationary prediction, we find that the high odd peak pattern of inflationary peaks is a unique prediction of inflation with a near BBN baryon content. Thus, the locations and relative peak heights can be used to "prove" the inflationary paradigm. On the other hand, this signature relies on the baryon drag effect, which may be small and difficult to observe in some exotic inflationary models. Moreover, inflation does not preclude the presence of isocurvature perturbations (Linde 1985; Yokoyama \& Suto 1991). Disproof of inflation along these lines is more difficult - a common problem in cosmology!

\section{ROBUSTNESS OF THE CURVATURE MEASUREMENT}

Acoustic oscillations in the CMB provide an interesting and unique opportunity to measure the curvature of the universe. Features in the spectrum supply standard rulers with which to make a classical test of the geometry (Doroshkevich et al. 1978; Sugiyama \& Gouda 1992; Kamionkowski, Spergel, \& Sugiyama 1994; Hu \& White 1996). If the inflationary scenario is correct, the curvature can be measured to a few percent essentially from the location of the first acoustic peak. However, is the curvature measurement robust to changes in the underlying model? Clearly, the location of the first peak does not contain enough information. Isocurvature models predict peak locations with a different relation to the sound horizon. Radical changes in the baryon content from its BBN value and the thermal history from standard recombination can shift their positions. What additional information is required to ensure that the curvature measurement is robust?

\subsection{Angular Diameter Distance}

It is instructive first to review the general case for measuring the curvature by the angular diameter distance test. For

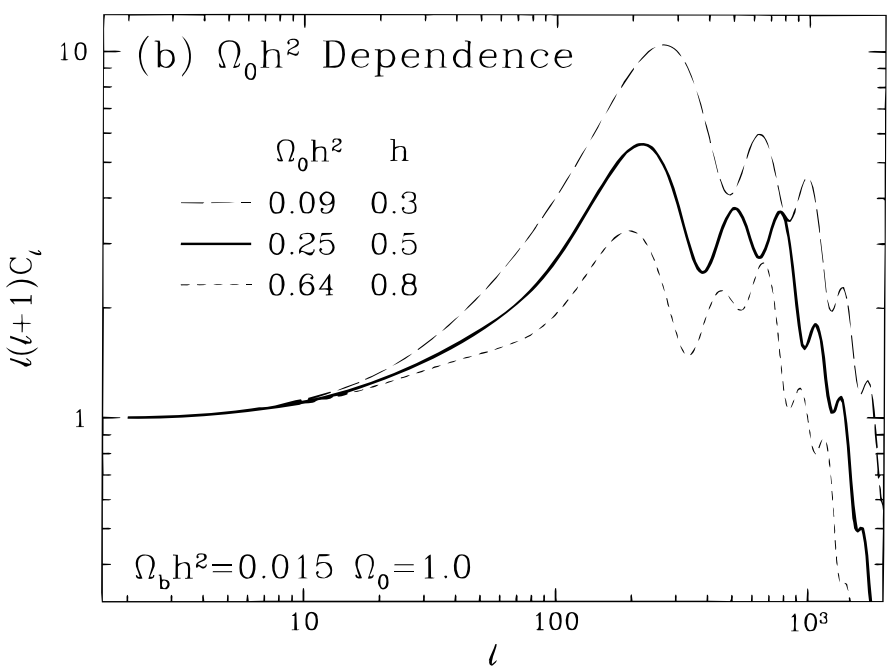

FIG. 10.-Baryon drag in the inflationary model. Baryon drag enhances the compressional, here odd, acoustic peaks. (a) Although diffusion damping at small scales coupled with the intrinsically small baryon drag effect in low $\Omega_{b} h^{2}$ models hides the effect, the third peak is clearly anomalously high in all but the most extreme case $\Omega_{b} h^{2}=0.0025$, which is in clear violation of BBN constraints. (b) Lowering $\Omega_{0} h^{2}$ also reduces the effect by reducing the potential fluctuation $\Psi$. 
definiteness and simplicity, let us take the inflationary example. Here the acoustic spectrum in $k$ is purely a function of $\Omega_{b} h^{2}$ and $\Omega_{0} h^{2}$. The implied fluctuations on the last scattering surface are viewed as anisotropies on the sky today via the projection

$$
l_{\text {feature }}(K, \Lambda)=k_{\text {feature }} r_{\theta}(K, \Lambda),
$$

where the comoving angular diameter distance to the last scattering surface is

$$
r_{\theta}(K, \Lambda)=|K|^{-1 / 2} \sinh \left[|K|^{1 / 2}\left(\eta_{0}-\eta_{*}\right)\right]
$$

for $K<0$ negatively curved universes. For $K>0$, merely replace $\sinh \rightarrow \sin$. The functional dependence arises since $\eta_{0}\left(K, \Lambda, \Omega_{0} h^{2}\right)$ and $\eta_{*}\left(\Omega_{0} h^{2}, \Omega_{b} h^{2}\right)$ and we are considering $\Omega_{0} h^{2}$ and $\Omega_{b} h^{2}$ fixed for now. In general, negative curvature moves the acoustic features to smaller angles since a fixed physical scale then subtends a smaller angle on the sky. A cosmological constant moves features to larger angles due to a decrease in the current horizon size from the rapid expansion.

In Figure 11, we display an example. By holding $\Omega_{b} h^{2}$ and $\Omega_{0} h^{2}$ constant, the acoustic features are fixed in physical space and the shift in $l$ is entirely due to the projection. Shifting the canonical $\Omega_{0}=1.0$ model by the angular diameter distance scaling of an $\Omega_{0}=0.1$ model, we see that equation (40) accurately accounts for the effect. Notice that $K$ and $\Lambda$ are degenerate with respect to the acoustic signature. One cannot in principle measure them independently from these features. The degeneracy is broken at larger angular scales by the ISW effect (HSb). Moreover, for realistic $\Omega_{0}, \Lambda$ introduces little ambiguity in the curvature measurement.

It is clear that either the peaks or the damping tail can be used as standard rulers to probe the curvature in this simple case. We shall now consider the benefits and drawbacks of each in the more general setting where the model for the perturbations and the background are not known a priori. We then turn to what can be learned about the model for the perturbations and how this additional information can be used to refine the measurement of the curvature.

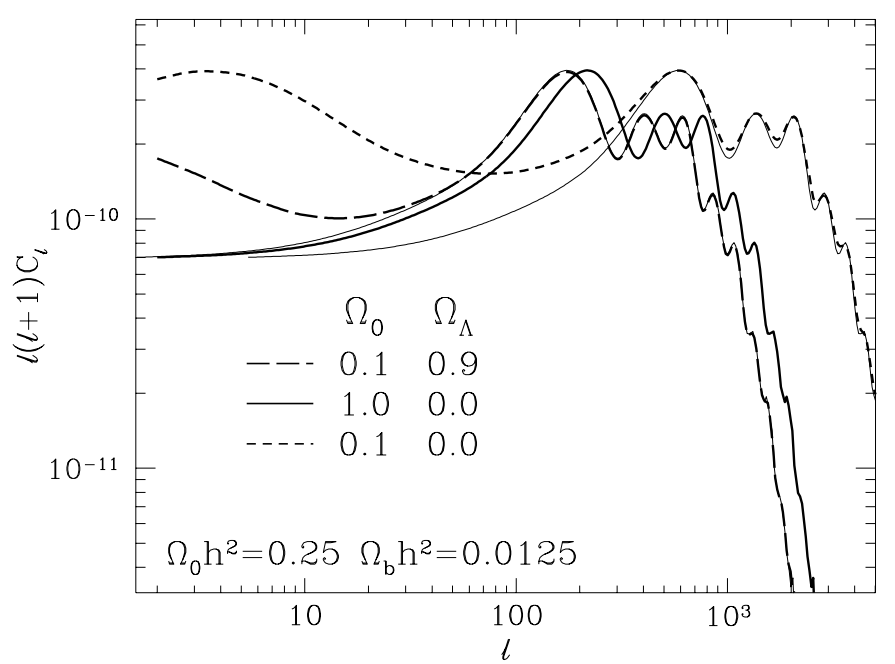

FIG. 11.-Standard rulers and the angular diameter distance. Acoustic features in the CMB, in particular the peak locations and the damping tail, act as standard rulers with which a measurement of the curvature can be made. Thin solid lines represent the $\Omega_{0}=1, \Lambda=0$ calculation scaled in $l$ to account for the projection in the $\Omega_{0}=0.1$ open and $\Lambda$ models.

\subsection{Acoustic Peaks}

The physical scale associated with the acoustic peaks is the sound horizon at last scattering. Unfortunately, however, the exact relation between the peak locations and the sound horizon typically varies by a factor of 2 depending on the model. In this section, we discuss a how the peak spacing can be used as a measurement of the spatial curvature, which is far less dependent on the model for the fluctuations.

As discussed in $\S 3.1$, once the source has switched off inside the horizon the acoustic peaks follow a phase-shifted harmonic series. The key point is that regardless of the phase, the peaks are spaced by $k_{A}=\pi / r_{s}$. Hence, if the sound horizon at last scattering is known, the spacing provides us with an angular diameter distance test of the curvature. In making this measurement, the higher peaks form a better probe than the lower peaks, since the latter can be shifted due to residual driving effects and metric fluctuations between last scattering and the present. The peak spacings $\Delta l$ for the models of Figure 7 are shown in Table 1 and should be compared with the prediction $\Delta l=l_{A} \equiv$ $k_{A} r_{\theta}$. As can be seen, the spacing becomes more regular and approaches the expected value after the first peak separation $l_{2}-l_{1}$. In the inflationary case shown here, baryon drag suppresses the even peaks and distorts their locations. For best accuracy, one should employ the spacings of the prominent peaks for the test, e.g., $l_{A}=\left(l_{3}-l_{1}\right) / 2$ in the inflationary case. In general, three peaks will be necessary to assure accuracy of the test.

How well can this test measure the curvature given realistic uncertainties in $\Omega_{b} h^{2}$ and $\Omega_{0} h^{2}$ through $h$, which affect the physical scale of the sound horizon at last scattering (see Fig. 12)? If the baryon content is near or less than the BBN limit $\Omega_{b} h^{2} \lesssim 0.05$, it has only a small effect since the photons dominate the fluid at last scattering. The Hubble constant has a relatively larger effect but even so a measurement of a peak spacing $l_{A} \sim 290$ would require $\Omega_{0}+\Omega_{\Lambda} \gtrsim$ 0.7 if $0.4 \leq h \leq 0.8$. Note that the dependence on the Hubble constant becomes weaker as it increases to make the universe more matter dominated at last scattering.

The main drawback of this method is that it may be difficult to apply in models where the forced effects continue well after horizon crossing. In this case, the peak spacing may not become regular until the higher peaks. Near the diffusion scale, the power in the CMB fluctuations is exponentially damped. Foregrounds become more difficult to

TABLE 1

Peak Spacings $\Delta l$ for the Models of FIGURE 7

\begin{tabular}{ccccc}
\hline \hline Model & $1-2$ & $2-3$ & $3-4$ & $4-5$ \\
\hline Inf $\ldots . . . \ldots$ & 288 & 259 & 297 & 277 \\
HDM..... & 204 & 282 & 272 & 295 \\
Tex ........ & 221 & 286 & 287 & 285 \\
Axi ......... 204 & 295 & 275 & 298 \\
\hline
\end{tabular}

NoTE-The spacing between peaks for the suite of models in Fig. 7 with the same background parameters $\Omega_{0}=1, \Omega_{b}=0.05, h=0.5$, and standard recombination. The spacing becomes more regular for the higher peaks and converges toward the expected value of 290 . The spacing yields a measure of the curvature of the universe that is nearly independent of the model for the fluctuations. 

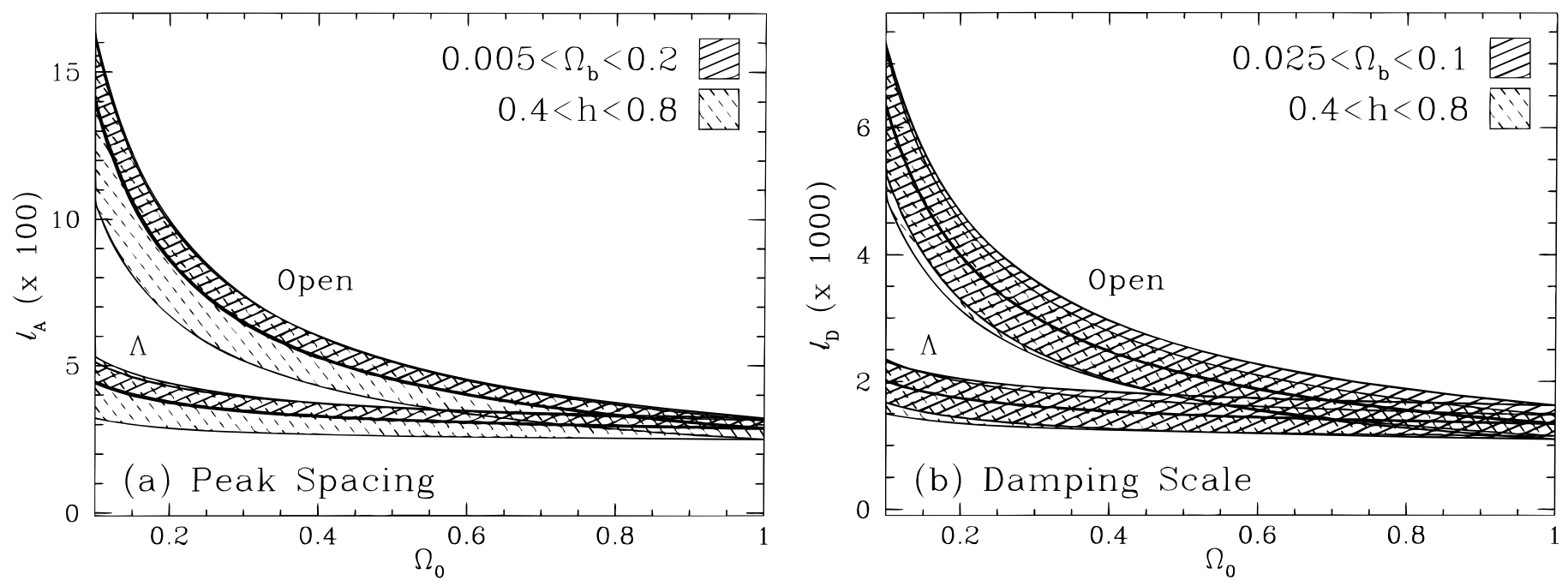

FIG. 12.-Peak spacing and damping scale as a function of $\Omega_{0}$. Even allowing for uncertainties in the baryon content (solid shading, $h=0.5$ ) and Hubble constant (dashed shading, $\Omega_{b} h^{2}=0.0125$ ), open models with $\Omega_{0} \lesssim 0.5$ can be distinguished from flat $\left(\Omega_{0}+\Omega_{\Lambda}=1\right) \Lambda$ models through either scale. The damping scale is entirely independent of the model for the fluctuations but may be more difficult to measure than the peak spacing.

subtract on these smaller scales and gravitational lensing by large-scale structure (see, e.g., Seljak 1996) can wash out the oscillations to some extent. It is even possible that stochastic metric perturbations would leave the spectrum with no distinct peaks (see $\S 2.6$, Magueijo et al. 1996). In these cases, we must turn to the damping tail to measure the curvature.

\subsection{Damping Tail}

The diffusion damping length provides a standard ruler for the curvature measurement that is the most robust against changes in the model for the fluctuations (see also $\mathrm{Hu} \&$ White 1996). As discussed in $\S 3.4$, its location is dependent only on background parameters. However, it is more sensitive to changes in $\Omega_{b} h^{2}$ and the thermal history due to its dependence on the Compton mean free path at last scattering. Figure $12 b$ displays the location of the damping tail $l_{D}$ defined as the multipole number at which the acoustic effect drops by $e^{-2}$ in power. It is related to the damping wavenumber equation (31) by the simple projection of equation (39). Here the thermal history has been fixed to follow standard recombination. By applying reasonable constraints on the other parameters, the damping scale becomes a sensitive probe of the curvature. Even allowing for a factor of 4 uncertainty in $\Omega_{b} h^{2}$, an open model of $\Omega_{0} \lesssim 0.5$ can be distinguished from a flat $\Omega_{0}$ $+\Omega_{\Lambda}=1$ universe. An additional uncertainty arises from the Hubble constant, which changes the age of the universe and expansion rate at last scattering and hence the diffusion scale. In Figure 12, we show the uncertainty from $h$ if $\Omega_{b} h^{2}$ is fixed by BBN. Again open universes with $\Omega_{0} \lesssim 0.5$ can be distinguished from the flat cases for reasonable values of $h$, amounting to a factor of 4 ambiguity in $\Omega_{0} h^{2}$.

There is one caveat to this proposal for measuring the curvature. It must first be established that the damping tail is indeed due to photon diffusion and not due to some intrinsic falloff in the source or secondary effect between recombination and the present. To test this assumption, one must measure the shape of the damping tail. Diffusion damping leads to a near exponential falloff in the anisotropy rather than the power-law behavior expected in the alternate possibilities. Thus, it will be necessary to follow the damped oscillations into the diffusion regime to estab- lish its exponential character. Even in a flat universe the damping tail is at arcminute scales. Detailed measurements of the primary signal may or may not be observationally feasible due to possible foreground and secondary effects, most notably the ISW effect. Models with large small-scale metric fluctuations may also have their effective temperature at last scattering dominated by baryon drag rather than acoustic oscillations. This too would mask the exponential signature. Of course, if acoustic peaks in the angular power spectrum are also measured, then it is almost assured that a break in the acoustic spectrum is due to diffusion.

\subsection{Exotic Baryon Content}

Thus far, we have mainly considered the robustness of the curvature measurement to exotic sources of gravitational perturbations. It is also possible that the background itself exhibits an exotic nature. In particular, we have been implicitly assuming that the baryon content is within a factor of several of its BBN value and the thermal history follows standard recombination. Let us now consider how the possibility of exotic background properties may be handled, beginning with the baryon content.

If the baryon content is far from the BBN prediction of $\Omega_{b} h^{2} \approx 0.01-0.02$, but known, the approach to measuring the curvature is unchanged since both the sound horizon and damping scale are known as well. It may be the case, however, that the prediction is violated by observations that do not then constrain $\Omega_{b} h^{2}$ sufficiently. If it is low, the peak spacing still provides a good measurement of the curvature. In this limit, the sound horizon at a fixed redshift becomes independent of the baryon content and the only variation with $\Omega_{b} h^{2}$ is a weak dependence on the redshift of last scattering (see Fig. 13a). However, the peaks must still be measured for this test to work. With a low baryon content, the Compton mean free path and hence the diffusion length at last scattering increases making the higher peaks difficult to observe. The ratio of the damping tail to the peak spacing $l_{D} / l_{A}$ provides a rough model independent estimate of the number of peaks that are potentially observable and is displayed in Figure 13a (cf. Fig. 5). Two peaks and hence the peak spacing is likely to be observable as long as $\Omega_{b} h^{2} \gtrsim 0.001$, which is close to the stellar mass density 

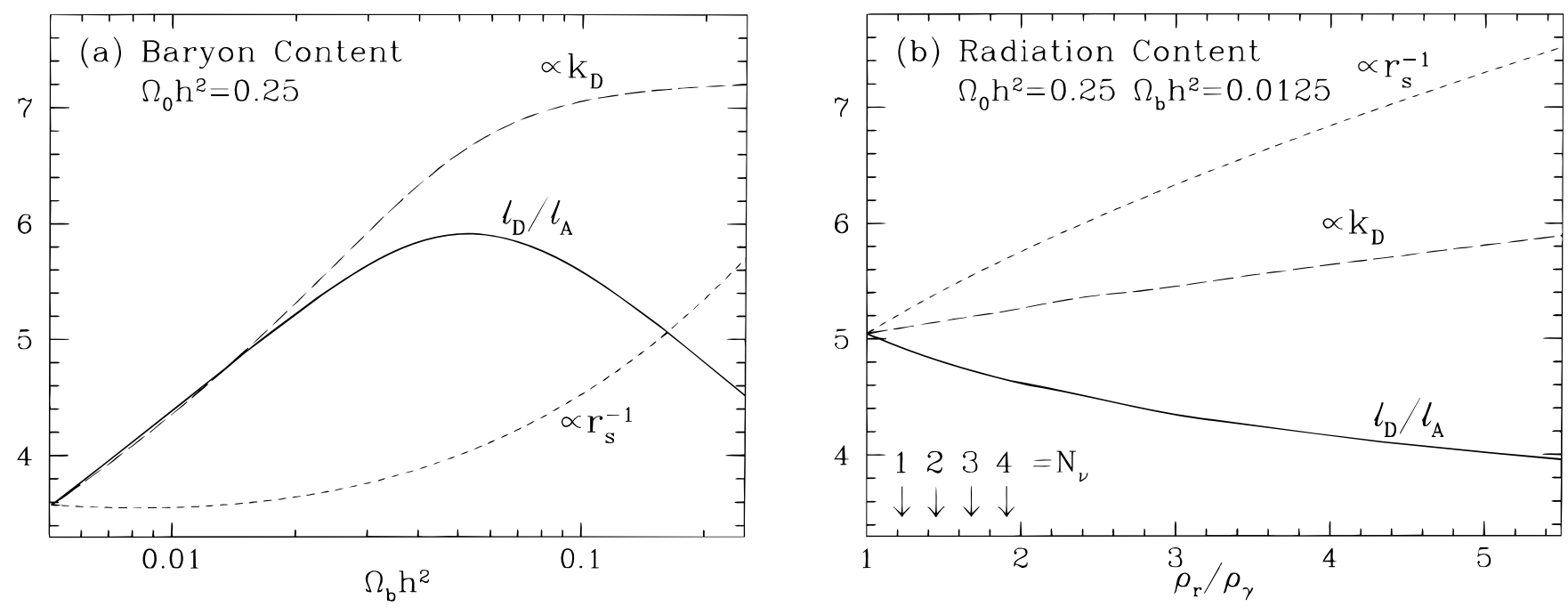

FIG. 13. - Exotic baryon and radiation content. (a) The sound horizon that forms the basis of the peak spacing test is independent of $\Omega_{b} h^{2}$ for values near BBN or lower, whereas the damping tail is insensitive to high $\Omega_{b} h^{2}$. In either limit a robust test exists. The ratio of tail to peak spacing $l_{D} / l_{A}$ can detect an exotic baryon content. (b) Changing the radiation content, e.g., by altering the number of relativistic neutrinos $N_{v}$, affects the expansion rate and thus the two physical scales weakly. Cases extreme enough to affect the scales significantly can also be distinguished by $l_{D} / l_{A}$.

$\Omega_{\text {stars }} \approx 0.004$ for a reasonable Hubble constant. Thus, the peak spacing should provide a test of the curvature even under the most extreme conditions. On the other hand, a curvature measurement from the damping tail alone could fall victim to this exotic possibility. However, the severely truncated acoustic spectrum in this case should prevent such a misinterpretation of the position of the damping tail.

In the case of an extremely high baryon content, the situation is reversed. The damping tail now provides the more robust estimate. Since the effects of a delay in last scattering and a decrease in the Compton mean free path tend to cancel, the physical scale is nearly independent of the baryon content for high values (see Fig. 13). On the other hand, if the baryon content is raised more than a factor of 5 over the BBN value, the sound speed and hence the sound horizon at last scattering decreases. Thus, the peak spacing alone is an unreliable test of the curvature if the baryon content is extremely high $\left(\Omega_{b} h^{2} \gtrsim 0.06\right)$ but unknown.

Is there a model-independent measure of the baryon content that can test for anomalous values? It should be clear from the discussion above that the ratio of the damping tail to peak spacing is highly sensitive to $\Omega_{b} h^{2}$. It is also a function of $\Omega_{0} h^{2}$, but since $\Omega_{0}$ must be consistent with the inferred curvature only uncertainties in the Hubble constant enter if $\Omega_{\Lambda}=0$. The ratio is double valued so that its measurement would allow both a high and low baryon content solution (see Fig. 13a). As we shall see in $\S 5.6$, baryon drag provides a distinction between the two extremes from a measurement of the relative heights of the peaks. In summary, an exotic baryon content is detectable and does not present a problem for the curvature measurement if both the peak spacing and damping tail can be measured.

\subsection{Exotic Thermal History}

Exotic thermal histories are another possibility. By thermal history, we refer to both the thermodynamics of the expansion and the ionization history. Massive decaying particles can create an epoch of matter domination before last scattering, which changes the age of the universe at last scattering and hence both the sound horizon and the diffu- sion scale (White, Gelmini, \& Silk 1995; Bardeen, Bond, \& Efstathiou 1987a). A less exotic example of the latter effect is provided by any model that changes the epoch of equality.

Let us take the a simple but illustrative example of a change in the energy density in relativistic species. This can arise, for example, by changing the number of relativistic neutrinos or their temperature. In Figure $13 b$, we plot the sound horizon, damping scale, and the ratio between the two as a function of the fractional increase in the radiation density $\rho_{r} / \rho_{\gamma}$. The standard thermal history with three relativistic neutrinos predicts $\rho_{r} / \rho_{\gamma}=1.68$. Raising the radiation content increases the expansion rate and thus decreases both the sound horizon and diffusion scale at last scattering. Its effects are relatively weak unless the standard prediction grossly underestimates the radiation content. Because the diffusion scale is essentially the geometric mean of the horizon and Compton mean free path, it is a weaker function of the radiation content. Hence, if the universe really has a sufficiently exotic history to change the two physical scales at last scattering, it should be detectable in the ratio of tail location to peak spacing $l_{D} / l_{A}$.

The use of $l_{D} / l_{A}$ as a probe of the thermal history is especially powerful for models that delay last scattering significantly. This might occur due to energy injection near $z \sim 10^{3}$ from particle decays or nonlinear fluctuations. Since the Compton mean free path grows as the universe expands, the diffusion scale can approach the horizon scale (see Fig. 14). Here full ionization is assumed until a redshift $z_{*}$, when recombination is taken to occur instantaneously. Instant recombination is not realistic; however, it provides an upper limit on $l_{D}$. The ratio of $l_{D} / l_{A}$ in Figure 14 then measures the delay in recombination. Under the instantaneous recombination assumption it corresponds to the number of observable peaks. The actual observable value for any given model will be lowered in a manner dependent on the details of recombination.

Even if recombination never occurred, the Compton mean free path will eventually reach the horizon size due to dilution in the electron number density from the expansion. At this point the universe is effectively transparent and the photons free stream to the present. Since by definition the 


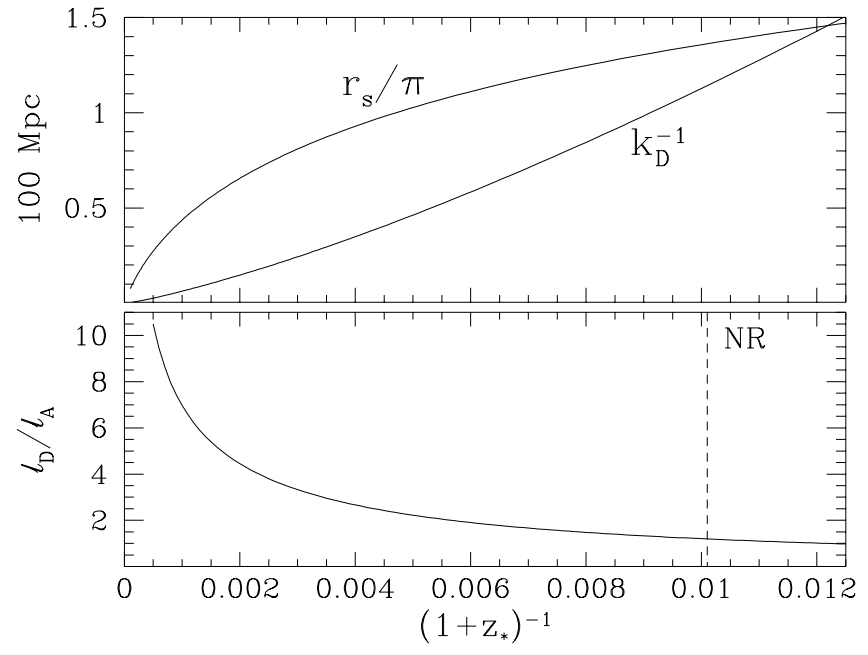

FIG. 14.-Exotic ionization history $\left(\Omega_{0} h^{2}=0.25, \Omega_{b} h^{2}=0.0125\right)$. We show the damping and peak separation scales in a model with instantaneous recombination at $z_{*}$. For a gradual recombination, a lower $l_{D} / l_{A}$ will always result. The diffusion scale at last scattering by definition approaches the sound horizon in the limit that no recombination (NR) occurred. Assuming instantaneous recombination, the ratio $l_{D} / l_{A}$ roughly corresponds to the number of observable peaks and can be used to discriminate against exotic ionization histories.

diffusion length roughly corresponds to the horizon scale at this epoch, $l_{D} / l_{A} \sim 1$ and no acoustic oscillations are apparent. Thus, recombination is a necessary condition for the acoustic signature to be observable. In fact for practical purposes, we require at least two observable peaks. For $\Omega_{0} h^{2}=0.25$ and $\Omega_{b} h^{2}=0.0125$, this translates to recombination by $z_{*}=175$. For comparison, late reionization after standard recombination does not destroy the acoustic signal unless the optical depth through the reionized epoch is greater than unity: $z_{\text {reion }} \gtrsim$ $100\left(\Omega_{0} h^{2} / 0.25\right)^{1 / 3}\left(\Omega_{b} h^{2} / 0.0125\right)^{-2 / 3}$.

Although there is not enough information in the one number $l_{D} / l_{A}$ to reconstruct all possible exotic thermal histories, it is possible to determine that some exotic model is necessary if $l_{D} / l_{A}$ is not $\approx 4-5$. Further work on the damping tail is necessary before this constraint can be tightened ( $\mathrm{Hu} \&$ White, in preparation). In this case, we trade precise knowledge of the curvature for evidence that exotic physics is required in the early universe. On the other hand, if the deviation is known to occur due to a specific cause such as early energy injection or delays in equality, then the curvature can once again be obtained. Moreover, with this information from both the damping tail and the peaks, we cannot mistakenly infer a value of the curvature due to exotic baryon content or thermal history.

\subsection{Toward Reconstructing the Model}

The previous sections considered constraints on the curvature that could be made with minimal knowledge of the model for the perturbations. However, we would ideally like to learn as much as possible about both questions from the CMB spectrum. In this section, we shall outline a program for measuring the curvature by first establishing the basic model for structure formation. Model properties such as the relative peak heights simplify the task of measuring the curvature since they provide extra clues. As noted in $\$ \S 5.2-5.3$, this may be the only way to proceed if it proves impossible to measure the higher acoustic peaks and damping scale.
We define the basic model possibilities by their dominant mechanism for forming anisotropies:

1. Inflation: correlated superhorizon curvature fluctuations. Although inflation may also produce isocurvature initial conditions, we class them separately.

2. Driven isocurvature (e.g., axionic and baryonic isocurvature, textures, etc.): photon-compensated initial conditions. We allow for the possibility that the first peak is obscured (see $\S 4.2$ ) especially in high baryon cases in which it is strongly suppressed with respect to the second peak.

3. Forced isocurvature (e.g., possibly strings): forces after but temporally correlated with horizon crossing.

4. Stochastic isocurvature (e.g., possibly strings): random subhorizon forces.

5. Reionized: any of the above in which the diffusion length and horizon length coincide, e.g., fully ionized models.

Each of these models can have anomalously high (low) baryon content defined as $\Omega_{b} h^{2}$ significantly larger (smaller) than the big bang nucleosynthesis $(\mathrm{BBN})$ value of $1 \%-2 \%$. They can also be low in dark matter (CDM) such that $\Omega_{0} \sim \Omega_{b}$. It is possible, though unlikely, that the model may be a mixture of "inflation" (as defined above) and driven isocurvature scenarios.

For definiteness, we hereafter discount the possibility of an exotic thermal history as treated in $\S 5.5$, except for the case of late reionization which is plausible in many models. Furthermore, we assume that the Hubble constant and $\Lambda$ are sufficiently constrained to make the confusion they introduce to the curvature measurement irrelevant (see, e.g., Fig. 12). We, however, relax the BBN constraints on $\Omega_{b} h^{2}$ as this can lead to a qualitatively distinct acoustic pattern.

There are four tests that we can apply to fix the model and the curvature, based on the acoustic signatures discussed in $\S 3$.

1. Peak positions test: measure the locations and spacings between the peaks in $l$. Three useful items can be extracted from this test:

Peak ratios.- Ratios probe the nature of the model. We define the distinguishing feature of a "cosine" series as a ratio of third to first peaks of $l_{3} / l_{1} \approx 3-4$ and that of a "sine" of $\approx 5$. Note that a true sine oscillation with missing first peak might be observationally classed as a cosine.

Peak spacings.-If they are regular, they provide an angular diameter distance test. The physical scale corresponds to $k_{A}=\pi / r_{s}$ if both compressional and rarefactional peaks are measured or $2 \pi / r_{s}$ for the compressional ones.

Peak-to-spacing ratio.- Since the peak spacing is fixed by the sound horizon, its ratio with respect to the first peak location provides a sensitive probe of the model. Inflation predicts $l_{1} / l_{A} \approx 0.7-0.9$.

2. Relative height test: determine if any of the peaks are anomalously high with respect to a smooth underlying spectrum. Heights probe the baryon content and forcing mechanism.

3. Tail-to-spacing test: measure the ratio of tail to peak spacing $l_{D} / l_{A}$. If the peak spacing is regular, the ratio measures the baryon content $\Omega_{b} h^{2}$ (and/or identifies exotic thermal histories).

4. Damping tail test: measure the shape and absolute location of the damping tail as described in $\S 5.3$. The shape confirms its acoustic nature and the location provides an angular diameter distance test. 
As we shall see, in most cases not all four tests are necessary. In contrast to $\S 5.3$, we shall here adopt the philosophy that information from the damping tail only be used if all other tests are ambiguous. If the baryon content is assumed to be known from nucleosynthesis, most of the tests involving the damping tail are generally unnecessary. On the other hand, the damping scale ideally should still be measured since it provides a valuable consistency check on both the baryon content and the thermal history assumption. Let us outline the program as a flow chart. It starts with the suite of peak position tests. There are four possibilities, as outlined in $\S \S 5.6 .1-5.6 .4$.

\subsubsection{The Peak Ratios Form a Cosine Series}

Apply the relative heights test.

1. High odd peaks forming a smooth sequence: inflation with BBN or high $\Omega_{b} h^{2}$. Tail-to-spacing fixes $\Omega_{b} h^{2}$. Spacing measures the curvature.

2. High odd peaks with anomalously high first peak: high $\Omega_{b} h^{2}$ driven isocurvature with first peak obscured. Tailto-spacing fixes $\Omega_{b} h^{2}$. Spacing measures curvature.

3. High even peaks: tuned forced isocurvature with near $\mathrm{BBN} \Omega_{b} h^{2}$. Spacing measures the curvature.

4. Monotonic: $\mathrm{BBN}$ or lower $\Omega_{b} h^{2}$. Spacing measures curvature.

a. Peak-to-spacing ratio $<1$ : low CDM inflation. Tailto-spacing can also measure $\Omega_{b} h^{2}$.

b. Peak-to-spacing ratio $>1$ : driven isocurvature with obscured first peak. Tail-to-spacing can also measure $\Omega_{b} h^{2}$.

\subsubsection{The Peak Ratios Form a Sine Series}

Apply the relative heights test.

1. High even peaks: driven isocurvature model with high $\Omega_{b} h^{2}$. Tail-to-spacing fixes $\Omega_{b} h^{2}$. Spacing or damping tail measures curvature.

2. High second peak: driven isocurvature model with BBN $\Omega_{b} h^{2}$ or lower. Spacing measures curvature. Tail-tospacing can also measure $\Omega_{b} h^{2}$.

3. Monotonic: high $\Omega_{b} h^{2}$, high CDM inflation. Tail-tospacing fixes $\Omega_{b} h^{2}$ and half-spacing determines curvature.

\subsubsection{Neither Cosine nor Sine}

1. Peaks follow phase-shifted harmonic: Coherent and tuned superposition of inflationary and driven isocurvature (cf. $\S 3.1$ ). Tail-to-spacing fixes $\Omega_{b} h^{2}$. Spacing measures curvature.

2. Peaks follow a cosine harmonic with gaps $1: 3: 4: 5$ : High $\Omega_{b} h^{2}$, relatively high $\Omega_{0} / \Omega_{b}$ inflation. Tail-to-spacing fixes $\Omega_{b} h^{2}$. Spacing of higher peaks or half-spacing across the gap measures curvature. Damping tail also measures curvature.

3. First few peaks irregular followed by a regular series. Forced isocurvature model with horizon crossing effects and BBN or lower $\Omega_{b} h^{2}$. Peak spacing measures curvature. Tail-to-spacing ratio can also measure the $\Omega_{b} h^{2}$.

4. Somewhat irregular peaks offset by smooth function (e.g., Fig. 3). Forced isocurvature model with effects well after horizon crossing and high $\Omega_{b} h^{2}$. Take average value of half-peak spacing. Tail-to-(average)-spacing fixes $\Omega_{b} h^{2}$. Average spacing measures curvature.

5. Random locations: Forced isocurvature model with rapidly varying metric well after horizon crossing. Tail-tospacing ratio constrains $\Omega_{b} h^{2}$. Damping tail constrains curvature.
5.6.4. No Peaks

Apply the damping tail test.

1. Exponential falloff: Stochastic isocurvature model. Location of damping tail measures the curvature if $\Omega_{b} h^{2}$ is known.

2. Power-law falloff: Reionized or stochastic isocurvature model with peak source amplitude far inside horizon and high $\Omega_{b} h^{2}$. No robust constraints on the curvature are possible.

Thus, in all but the last case the acoustic signature constrains the curvature. If the baryon content can also either be measured from the signature itself or is known from external constraints such as BBN, highly accurate measurements of the curvature are possible. Once the basic nature of the model and background is determined through this program, detailed modeling of the fundamental source for the fluctuations that formed large-scale structure may begin.

\section{CONCLUSIONS}

We have generalized the formalism of HSa to include backreaction effects and examined the uniqueness and robustness of acoustic signatures in the CMB. By clarifying the role of compensation and feedback in the evolution of fluctuations, we have shown that the phase of the oscillation, and hence the ratios of peak locations, distinguishes inflation from "typical" isocurvature models. Specifically, two robust tests are the ratio of third to first peaks and first to peak spacing. Our analysis also provides a better understanding of the structure of the peaks in these models. However, since it is possible to imagine isocurvature models that are tuned to mimic the inflationary pattern of peaks, we have stressed the importance of baryon drag, which allows us to distinguish compressions from rarefactions in potential wells. This can help lift the confusion between adiabatic models and these contrived isocurvature models. Although the level of drag does not make this distinction clear for all possible baryon densities, it is observable for the value predicted by big bang nucleosynthesis. Further understanding of the diffusion damping of anisotropies will allow us to extend the lower limit of baryon densities for which we can distinguish the rarefaction and compression peaks.

We focus on the importance of the damping tail as a measure of spatial curvature that is independent of the model for structure formation, and discuss the robustness of curvature measurements from the CMB. Even in the case where no acoustic peaks are seen (e.g., possibly string models) the damping scale can be estimated under mild assumptions about the thermal history and baryon content. A more precise test is possible if the acoustic peaks are regularly spaced as indeed expected of models without extreme behavior at small scales. Either approach allows one to infer the physical scale of the acoustic feature(s). Its projection onto the sky allows us to perform a classical angular diameter distance test to determine the curvature of the universe. In the process of carrying out these tests of the curvature, the general nature of the model for the fluctuations can be reconstructed, as well as the baryon content of the universe.

All of these studies focus on the tale told by the CMB spectrum taken as a whole. In particular, the acoustic pattern, which arise from forced oscillations in the photonbaryon fluid before recombination, leaves a distinct signa- 
ture from which we may begin to reconstruct the cosmological model.

We would like to acknowledge useful conversations with J. Bahcall, P. Ferreira, A. Kosowsky, and A. Stebbins. We would also like to thank R. Crittenden and A. de Laix for supplying power spectra from their calculations of the texture and HDM isocurvature models, as well as N. Sugiyama for use of his Boltzmann code. W. H. was supported by grants from the NSF and W. M. Keck foundation.

\section{APPENDIX}

\section{CAUSALITY AND COMPENSATION}

In this Appendix, we clarify the role of causality in limiting the behavior of fluctuations outside the horizon and its dependence on gauge. We show that only inflation can correlate the curvature fluctuations above the horizon assuming general relativity is the correct description of gravity. Moreover, in the main text we employed only the mechanism of compensation, i.e., the response of the photon-baryon fluid to a source, and not the full causal constraints that limit the behavior of the source as well. This proved sufficiently powerful to produce the distinctions in the acoustic signature under the additional assumption that the compensation is provided by the photons. In this case, the feedback from the photon self-gravity produces the key ingredient in making many of the signatures robust. Since in the standard FRW model, the universe is radiation dominated until near recombination, this additional assumption is automatically satisfied. ${ }^{1}$ There are, however, exotic models where this assumption is not satisfied. For example, a decaying massive particle could cause the universe to undergo a period of matter domination before recombination. For this kind of situation, we need to examine the general case of compensation and additional causal constraints on the model.

Causality implies initial compensation in density fluctuations above the horizon since the stress-energy tensor is conserved. Heuristically, the conservation law implies that changes in energy density at any location arise from "flows" of energy density current across surfaces, or from displacements of fluid elements. Since fluid elements cannot be displaced "beyond" the horizon, this severely constrains the behavior of fluctuations at $k \eta \ll 1$. However, because the stress-energy tensor obeys covariant conservation, the exact form of causal constraints on the density perturbation depends on the representation of the metric, i.e., the gauge.

We have seen in equation (1) that density perturbations can also change due to the "stretching" effects from changes in the metric. A clever choice of gauge can eliminate such effects. Let us examine the evolution of the density fluctuation in its local rest frame. Note that this does not coincide with hypersurfaces with zero bulk velocity for the total matter $V_{T}$ unless $g_{0 i}$ vanishes. In the literature, this has been called the comoving gauge (Bardeen 1980), velocity-orthogonal isotropic gauge (Kodama \& Sasaki 1984) and total matter gauge (HSb). It represents the metric fluctuations as

$$
\begin{aligned}
g_{00} & =-a^{2}(1+2 \xi Q), \\
g_{0 j} & =a^{2} V_{T} k^{-1} Q_{\mid j}, \\
g_{i j} & =a^{2}(1+2 \zeta Q) \gamma_{i j},
\end{aligned}
$$

where | represents a covariant derivative with respect to $\gamma_{i j}$ and recall $Q$ is the $k$ th eigenfunction of the normal mode decomposition. These quantities are related to their Newtonian counterparts by a gauge transformation $\tilde{x}^{\mu}=x^{\mu}+\delta x^{\mu}$. If the line element $d s^{2}$ is to remain unchanged (to first order in $\delta x$ ),

$$
\tilde{g}_{\mu \nu}=g_{\mu \nu}+g_{\alpha \nu} \delta x^{\alpha}{ }_{, \mu}+g_{\alpha \mu} \delta x_{, \nu}^{\alpha}-g_{\mu v, \alpha} \delta x^{\alpha} .
$$

A similar relation follows for the stress energy tensor $T_{\mu \nu}$ and relates the matter quantities. For densities, it is simpler to note that it arises from a combination of a shift in time slicing and the background density evolution: $\tilde{\delta}_{i} Q=\delta_{i} Q-\left(\dot{\rho}_{i} / \rho_{i}\right) \delta x^{0}$. These relations imply that from the Newtonian gauge, the rest frame is reached by the coordinate shift $\delta x^{\mu}=\left(V_{T} / k, \overrightarrow{0}\right) Q$, and the perturbation quantities are related by

$$
\begin{aligned}
\xi & =\Psi-\frac{\dot{V}_{T}}{k}-\frac{\dot{a}}{a} \frac{V_{T}}{k}, \\
\zeta & =\Phi-\frac{\dot{a}}{a} \frac{V_{T}}{k}, \\
\Delta_{T} & =\delta_{T}+3\left(1+w_{T}\right) \frac{\dot{a}}{a} \frac{V_{T}}{k},
\end{aligned}
$$

where $w_{i}=p_{i} / \rho_{i}$ and subscript the $T$ denotes quantities of the total fluid from a sum over the particle constituents. Here we have used the background evolution equation $\dot{\rho}_{i} / \rho_{i}=-3\left(1+w_{i}\right)(\dot{a} / a)$. Note that the velocity $V_{T}$ is the same in the Newtonian and rest frame gauges since its transformation properties depend on $\delta x^{i}$. The familiar quantity $\zeta$ is the curvature of the spatial hypersurfaces in this gauge (Bardeen 1980).

\footnotetext{
${ }^{1}$ Note that the universe need only be radiation dominated when the fluctuation was well outside the horizon, not at last scattering itself. This is of course not satisfied in models with significant reionization, but in this case acoustic oscillations are not observable anyway.
} 
Applying the gauge transformation equation (A3) to the Newtonian gauge equations or writing the Einstein equations in this gauge (see HSb, eq. [16]; Kodama \& Sasaki 1984, eq. [4.7]), we obtain the evolution equation for the curvature:

$$
\dot{\zeta}=\frac{\dot{a}}{a} \xi=-\frac{\dot{a}}{a} \frac{w_{T}}{1+w_{T}}\left(\frac{\delta p_{T}}{p_{T}}-\frac{2}{3} \Pi_{T}\right) .
$$

Unless $w_{T}=-1$ as in the case of the de Sitter phase, the rest frame curvature remains constant in the absence of isotropic (pressure) or anisotropic stress perturbations. The isotropic stress perturbation can be broken up into an adiabatic and nonadiabatic part:

$$
\frac{\delta p_{T}}{p_{T}}=c_{T}^{2} w_{T}^{-1} \Delta_{T}+\Gamma_{T},
$$

with the sound speed of the total fluid given by $c_{T}^{2}=\dot{p}_{T} / \dot{\rho}_{T}$.

The adiabatic pressure perturbation is related to the curvature fluctuation by a factor of $(k \eta)^{2}$ through the Poisson equation and hence is a negligible source for $(k \eta)^{2} \ll 1$. The generalized compensation law is therefore that component evolution must balance at $k \eta \ll 1$ to keep the rest frame curvature constant in the absence of nonadiabatic isotropic stress ("entropy") or anisotropic stress perturbations. Note that unlike the adiabatic isotropic stress, both of these sources are the same in any frame and admit no gauge ambiguity.

Since the continuity equation in this gauge is

$$
\frac{d}{d \eta}\left(\frac{\Delta_{i}}{1+w_{i}}\right)=-\left(k V_{i}+3 \dot{\zeta}\right),
$$

for each of the individual particle species, the rest frame density perturbation obeys an ordinary conservation law if these stresses are absent $(\dot{\zeta}=0)$. In other words, the number density of each of the particle constituents only changes through their bulk motion. Thus, we see that the causal constraint is simplest in the rest frame. Note that equations (A6) and(A4) imply that although density fluctuations can change purely due to an evolving equation of state, this does not affect the curvature fluctuation $\zeta$ above the horizon.

Now let us consider the source of curvature fluctuations from nonadiabatic pressure perturbations. The analysis also applies to anisotropic stress perturbations. The curvature fluctuation generated by pressure perturbations is of order $\delta p_{T} /\left(p_{T}+\rho_{T}\right)$. This fact is somewhat counterintuitive since the physical mechanism that converts a pressure fluctuation to a density fluctuation is the movement of matter, which is impossible beyond the horizon. Let us examine its qualitative origin. Causality constraints in Fourier space do not require no evolution for $k \eta \ll 1$. In physical space, motion of matter through length scales up to $\eta$ cause a suppressed evolution of the Fourier amplitude. A change in momentum density of the fluid is caused by the pressure gradient and generates a bulk velocity of order $(k \eta) \delta p_{T} /\left(p_{T}+\rho_{T}\right)$. This, then, forms a kinematic density fluctuation from the continuity equation (A6) of order $(k \eta)^{2} \delta p_{T} /\left(p_{T}+\rho_{T}\right)$ or a curvature fluctuation of order $\delta p_{T} /\left(p_{T}+\rho_{T}\right)$. Thus, the residual curvature fluctuation induced by motion of matter inside the horizon is generically of order $w_{T} /\left(1+w_{T}\right)$ times the pressure perturbation.

Causality implies that before matter has had a chance to move around, the universe must obey the isocurvature condition $\zeta=0$ or $\Delta_{T}=0 .^{2}$ If the condition is met by balancing perturbations in the different components of the fluid, a nonadiabatic pressure fluctuation generically arises,

$$
p_{T} \Gamma_{T} \equiv \delta p_{T}-c_{T}^{2} \delta \rho_{T}=\sum\left(c_{i}^{2}-c_{T}^{2}\right) \delta \rho_{i},
$$

if the equation of state of the balancing components differ. As we have seen, $\Gamma_{T}$ can produce a curvature fluctuation even at $k \eta \ll 1$. How does this possibility affect our arguments concerning the uniqueness of the inflationary spectrum? First, we need to relate the rest frame curvature to the Newtonian curvature. Employing the continuity equation (Kodama \& Sasaki 1984, eq. 4.7) in equation (A3), we find (Lyth 1985; Mukhanov et al. 1992)

$$
\zeta=\Phi+\frac{2}{3} \frac{1}{1+w_{T}}\left(\Phi+\frac{a}{\dot{a}} \dot{\Phi}\right)
$$

in the absence of anisotropic stress. If the equation of state is constant and $\Phi$ evolves as a power law, $\zeta \propto \Phi$. The two curvature fluctuations are comparable except in the degenerate case where $\zeta=0$ and

$$
\frac{\dot{\Phi}}{\Phi}=-\frac{\dot{a}}{a}\left[\frac{3}{2}\left(1+w_{T}\right)+1\right] \text {. }
$$

In fact, this is the case of the source-free decaying mode described by equation (14). The decaying mode thus carries no curvature perturbation in the rest frame. The other case in which $\zeta$ and $\Phi$ behave differently is through a change in the equation of state. For example, through the matter radiation transition $w_{T}$ goes from $\frac{1}{3} \rightarrow 0$. Although $\zeta$ remains constant in the absence of stress perturbations, $\Phi$ drops by a factor of 9/10 through the transition. For most purposes however, we can think of $\zeta$ and $\Phi$ as nearly interchangeable.

The mechanism by which nonadiabatic pressure perturbations generate curvature fluctuations is of course already implicitly incorporated in our analysis and is the cause of the curvature not being strictly zero outside at $k \eta \ll 1$ but merely

${ }^{2}$ More precisely, $\Delta_{T}=0$ aside from a $\zeta=0$ decaying mode (see eq. [A9]). 
suppressed. In the photon-dominated limit, only a small pressure fluctuation is needed to compensate a rather large density fluctuation in the source. Thus, the curvature generated by this effect is negligible. In the matter-dominated limit, pressure fluctuations cannot move a large amount of energy density as exhibited by the $w_{T} /\left(1+w_{T}\right)$ suppression. However, as the universe changes from radiation to matter domination a relatively significant curvature fluctuation can be generated.

The baryon isocurvature model provides a concrete example. In this case, the nonadiabatic pressure perturbation is

$$
\Gamma_{T}=-\frac{1-3 w_{T}}{1+w_{T}} S
$$

where $S=\delta\left(n_{b} / n_{\gamma}\right)=\delta_{b}-3 \Theta_{0}$ is the entropy fluctuation. Notice that the pressure fluctuation is small as long as the universe is radiation dominated $w_{T}=\frac{1}{3}$. The evolution equations may be exactly solved in the $k \eta \ll 1$ limit such that the Newtonian curvature is $\Phi=\frac{1}{8}\left(a / a_{e q}\right) S$ in the radiation-dominated limit and $\frac{1}{5} S$ in the matter-dominated limit (see HSb, eq. [27]). Notice that the curvature is a constant in the matter-dominated limit even outside the horizon. Around equality, pressure perturbations of order $S$ generate curvature fluctuations of the same order. Since in the matter-dominated limit, pressure is no longer effective, this curvature fluctuation is frozen in.

If one considers the evolution of a single $k$-mode, distinguishing between this and the inflationary case would be difficult since they both exhibit a constant curvature fluctuation above the horizon. In the standard scenario, this is not a problem since the matter-radiation transition cannot occur early enough without overclosing the universe $\left(\Omega_{0} h^{2} \rightarrow 1\right)$. Note that the curvature fluctuation must be constant well before horizon crossing for all observable scales in order to mimic inflation. However, it is possible with decaying massive particle scenarios for the universe to undergo a period of matter domination before the ordinary radiation-dominated epoch.

In this case, the spectrum of nonadiabatic pressure perturbations implied by causality serves to distinguish the model from inflation. Causality forbids spatial correlations in the spectrum of such perturbations, so $\left|\Gamma_{T}(k)\right|^{2}$ and hence $|\Phi(k)|^{2}$ are constant in $k$, i.e., white noise (or steeper if symmetries can be imposed), before horizon crossing. Notice that this agrees with the familiar result that the density perturbations generated by the motion of matter have a $\left|\Delta_{T}\right|^{2} \propto k^{4}$ tail for $k \eta \ll 1$ (Zel'dovich 1965; Robinson \& Wandelt 1996). This translates to a steeply rising spectrum of acoustic fluctuations compared with the inflationary case of an approximately scale-invariant spectrum $\left|\Phi\left(\eta_{i}, k\right)\right|^{2} \propto k^{-3}$. Thus, acoustic modes associated with a constant curvature perturbation outside the horizon generated from an isocurvature initial condition are both easily distinguished from inflation and observationally ruled out! The most general isocurvature spectrum with scale-invariant curvature perturbations at horizon crossing is $|\Phi(\eta, k)|^{2}=F(k \eta) k^{-3}$. With the requirement of white noise perturbations outside the horizon, $\Phi$ must grow as $\eta^{3 / 2}$ before horizon crossing. Since only models like these, with curvature fluctuations growing until horizon crossing, require observation of the acoustic signature to distinguish them from inflation, our assumption in the main text is justified. It is of course still possible that tuned effects around horizon crossing can mimic an inflationary spectrum in such a exotic scenario where the regulatory effects of photon feedback are absent. However, since both tuning and a drastic modification of the thermal history is necessary, we do not consider this possibility to be worth considering.

\section{REFERENCES}

Albrecht, A., Coulson, D., Ferreira, P., \& Magueijo, J. 1996, Phys. Rev. Lett., 76, 1413

Bardeen, J. M. 1980, Phys. Rev. D, 22, 1882

Bardeen, J. M., Bond, J. R., \& Efstathiou, G. 1987a, ApJ, 321, 28

Bardeen, J. M., Bond, J. R., Kaiser, N., \& Szalay, A. S. 1987b, ApJ, 304, 15

Bennett, D. P., Stebbins, A., \& Bouchet, F. R. 1992, ApJ, 399, L5

Bond, J. R., et al. 1994, Phys. Rev. Lett., 72, 13

Bucher, M., Goldhaber, A., \& Turok, N. 1995, Phys. Rev. D, 52, 3314

Chibisov, G. V. 1972, AZh, 49, 72

Coulson, D., Ferreira, P., Graham, P., \& Turok, N. 1994, Nature, 368, 27

Crittenden, R. G., \& Turok, N. G. 1995, Phys. Rev. Lett., 75, 2642

Davis, R., et al. 1992, Phys. Rev. Lett., 69, 1856; 70, 1733

de Laix, A. A., \& Scherrer, R. J. 1995, ApJ, 464, 539

Dodelson, S., Gates, E., \& Stebbins, A. 1995, preprint astro-ph/9509147

Doroshkevich, A. G., Zel'dovich, Ya. B., \& Sunyaev, R. A. 1978, Sov. Astron., 22, 523

Durrer, R., Gangui, A., \& Sakellariadou, M. 1996, Phys. Rev. Lett., 76, 579

Efstathiou, G., \& Bond, J. R. 1987, MNRAS, 227, 33P

Efstathiou, G., Bond, J. R., \& White, S. D. M. 1992, MNRAS, 258, 1P

Gorski, K., Ratra, B., Sugiyama, N., \& Banday, A. 1995, ApJ, 444, L65

Hu, W., \& Sugiyama, N. 1995a, ApJ, 444, 489 (HSa) 1995b, Phys. Rev. D, 51, 2599 (HSb) 1996, ApJ, 471, in press

Hu, W., Sugiyama, N., \& Silk, J. 1996, Nature, in press

Hu, W., \& White, M. 1996, A\&A, in press

Hu, Y., Turner, M. S., \& Weinberg, E. J. 1994, Phys. Rev. D, 49, 3830

Jaffe, A. H., Stebbins, A., \& Frieman, J. A. 1994, ApJ, 420, 9

Jungman, G., Kamionkowski, M., Kosowski, A., \& Spergel, D. N. 1995, Phys. Rev. Lett., 76, 1007

Kaiser, N. 1983, MNRAS, 202, 1169

Kamionkowski, M., Spergel, D. N., \& Sugiyama, N. 1994, ApJ, 426, L57

Kawasaki, M., Sugiyama, N., \& Yanagida, T. 1995, preprint, hep-ph/9512368

Knox, L., \& Turner, M. S. 1994, Phys. Rev. Lett., 73, 3347
Kodama, H. \& Sasaki, M. 1984, Prog. Theor. Phys. Supp., 78, 1 1986, Int. J. Mod. Phys., A1, 265

Kolb, E., \& Turner, M. S. 1990, in The Early Universe (New York: Addison-Wesley)

Liddle, A. R. 1995, Phys. Rev. D, 51, 5347

Liddle, A. R., \& Lyth, D. H. 1993, Phys. Rep., 231, 1

Linde, A. 1985, Phys. Lett. B, 126, 178

Lyth, D. H. 1985, Phys. Rev. D., 31, 1792

Ma, C. P., \& Bertschinger, E. 1995, ApJ, 455, 7

Magueijo, J., Albrecht, A., Coulson, D., \& Ferreira, P. 1996, Phys. Rev. Lett., 76, 2617

Mukhanov, V. F., Feldman, H. A., \& Brandenberger, R. H. 1992, Phys. Rep., 215, 203

Ostriker, J. P., \& Steinhardt, P. 1995, Nature, 377, 600

Peebles, P. J. E. 1987, Nature, 327, 310

Peebles, P. J. E., \& Yu, J. T. 1970, ApJ, 162, 815

Pen, U. L., Spergel, D. N., \& Turok, N. 1994, Phys. Rev. D, 49, 692

Robinson, J., \& Wandelt, B. 1996, Phys. Rev. D, 53, 618

Sachs, R. K., \& Wolfe, A. M. 1967, ApJ, 147, 73

Scott, D. \& White, M. 1995, Gen. Relativ. Gravitation, 27, 1023

Seljak, U. 1994, ApJ, 435, L87 1996, ApJ, 463, 1

Silk, J. 1969, ApJ, 151, 459

Sugiyama, N. 1995, ApJS, 100, 281

Sugiyama, N., \& Gouda, N. 1992, Prog. Theor. Phys., 88, 803

Turner, M. S., \& White, M. 1996, Phys. Rev. D, 53, 6822

Veeraraghavan, S., \& Stebbins, A. 1990, ApJ, 365, 37

Weinberg, S. 1972, Gravitation and Cosmology (New York: Wiley), 568

White, M., Gelmini, G., \& Silk, J. 1995, Phys. Rev. D, 51, 2669

White, M., Krauss, L. M., \& Silk, J. 1993, ApJ, 418, 535

Wilson, M. L. 1983, ApJ, 273, 2

Yamamoto, K., Sasaki, M., \& Tanaka, T. 1995, ApJ, 455, 412

Yokoyama, J., \& Suto, Y. 1991, ApJ, 379, 427

Zel'dovich, Ya. B. 1965, Adv. Astron. Astrophys., 3, 241 